\title{
Asymptotic Theory for the QMLE in GARCH-X Models with Stationary and Non-Stationary Covariates
}

\author{
Heejoon Han ${ }^{*} \quad$ Dennis Kristensen ${ }^{\dagger}$
}

JANUARY 2014

\begin{abstract}
This paper investigates the asymptotic properties of the Gaussian quasi-maximum-likelihood estimators (QMLE's) of the GARCH model augmented by including an additional explanatory variable - the so-called GARCH-X model. The additional covariate is allowed to exhibit any degree of persistence as captured by its long-memory parameter $d_{x}$; in particular, we allow for both stationary and non-stationary covariates. We show that the QMLE's of the parameters entering the volatility equation are consistent and mixed-normally distributed in large samples. The convergence rates and limiting distributions of the QMLE's depend on whether the regressor is stationary or not. However, standard inferential tools for the parameters are robust to the level of persistence of the regressor with $t$-statistics following standard Normal distributions in large sample irrespective of whether the regressor is stationary or not.
\end{abstract}

Keywords: Asymptotic properties, GARCH-X, persistent co-variate, quasi-maximum likelihood, robust inference.

\section{Introduction}

To better model and forecast the volatility of economic and financial time series, empirical researchers and practitioners often include exogenous regressors in the specification of volatility dynamics. One particularly popular model within this setting is the so-called GARCH-X model where the basic GARCH specification of Bollerslev (1986) is augmented by adding exogenous regressors to the volatility equation:

$$
y_{t}=\sigma_{t}(\vartheta) \varepsilon_{t},
$$

where $\varepsilon_{t}$ is the error process while $\sigma_{t}^{2}(\vartheta)$ is the volatility process given by

$$
\sigma_{t}^{2}(\vartheta)=\omega+\alpha y_{t-1}^{2}+\beta \sigma_{t-1}^{2}+\pi x_{t-1}^{2},
$$

${ }^{*}$ Department of Economics, Kyung Hee University. E-mail: heejoon@khu.ac.kr.

${ }^{\dagger}$ Corresponding author. Dep. of Economics, UCL, CREATES and IFS. E-mail: d.kristensen@ucl.ac.uk. 
for some observed covariate $x_{t}$ which is squared to ensure that $\sigma_{t}^{2}(\vartheta)>0$, and where $\vartheta=\left(\omega, \theta^{\prime}\right)^{\prime}$, $\theta=(\alpha, \beta, \pi)^{\prime}$, is the vector of parameters. The inclusion of the additional regressor $x_{t}$ often helps explaining the volatilities of stock return series, exchange rate returns series or interest rate series and tend to lead to better in-sample fit and out-of sample forecasting performance. Choices of covariates found in empirical studies using the GARCH-X model span a wide range of various economic or financial indicators. Examples include interest rate levels (Brenner et al., 1996; Glosten et al, 1993; Gray, 1996), bid-ask spreads (Bollerslev and Melvin, 1994), interest rate spreads (Dominguez, 1998; Hagiwara \& Herce, 1999), forward-spot spreads (Hodrick, 1989), futures open interest (Girma and Mougoue, 2002), information flow (Gallo and Pacini, 2000), and trading volumes (Fleming et al, 2008; Lamoureux and Lastrapes, 1990; Marsh and Wagner, 2005). More recently, various realized volatility measures constructed from high frequency data have been adopted covariates in the GARCH-type models with the rapid development seen in the field of realized volatility; see Barndorff-Nielsen and Shephard (2007), Engle (2002), Engle and Gallo (2006), Hansen et al. (2012), Hwang and Satchell (2005), and Shephard and Sheppard (2010).

While the GARCH-X model and its associated quasi-maximum likelihood estimator (QMLE) have found widespread empirical use, the theoretical properties of the estimator are not fully understood. In particular, given the wide range of different choices of covariates, it is of interest to analyze how the persistence of the chosen covariate influences the QMLE. As shown in Table 1, the degree of persistence varies a lot across some popular covariates used in GARCH-X specifications. The table reports log-periodogram estimates of memory parameter, $d_{x}$, and estimates of the first-order autocorrelation, $\rho_{1}$, for some time series used as covariates in the literature. For example, interest rate levels and bond yield spreads are highly persistent with estimates of $d_{x}$ being mostly larger than 0.8 and $\rho_{1}$ estimates close to unity, thereby suggesting unit root type behaviour. Meanwhile, realized volatility measures (realized variance) of various stock index and exchange rate return series are less persistent with estimates of $d_{x}$ ranging between 0.3 and 0.6 while the estimates of $\rho_{1}$ are relatively small and taking values between 0.64 to 0.88 ; formal unit root tests clearly reject unit root hypotheses for these time series. A natural concern would be that different degrees of persistence of the chosen covariates would lead to different behaviour of the QMLE and associated inferential tools.

We provide a unified asymptotic theory for the QMLE of the parameters allowing for both stationary and non-stationary regressors. In the stationary case, we do not impose any further restrictions on the dynamics of $x_{t}$. In the case of non-stationary regressors, on the other hand, we specifically model $x_{t}$ as an $I\left(d_{x}\right)$ process with $1 / 2<d_{x}<3 / 2$. This allows for a wide range of persistence as captured by the long-memory parameter $d_{x}$, including unit root processes $\left(d_{x}=1\right)$ but also processes with either weaker $\left(d_{x}<1\right)$ or stronger dependence $\left(d_{x}>1\right)$.

Our main results show that to a large extent applied researchers can employ the same techniques when drawing inference regarding model parameters regardless of the degree of persistence of the regressors. We first show that QMLE consistently estimates $\vartheta_{0}$ whether $x_{t}$ is stationary or not, but that its convergence rates and limiting distribution changes when $x_{t}$ is non-stationary. In particular, its distribution is mixed-normal in the non-stationary case. At the same time, we 
also demonstrate that the large sample distributions of $t$-statistics are invariant to the degree of persistence and always follow $N(0,1)$ distributions. This last limit result is due to the fact that in the computation of t-statistics, the QMLE's are normalized by estimators of the square root of its quadratic variation. In the stationary case, the estimated quadratic variation of the QMLE's converge towards a constant as is standard. On the other hand, in the non-stationary case, the limiting quadratic variation is random which leads to the distribution of the QMLE's to be mixed-normal. This self-normalization of the QMLE's when computing $t$-statistics removes any non-Gaussian component of the limiting distribution of the QMLE's and so the statistics converge towards $N(0,1)$ distributions even in the non-stationary case. As consequence, standard inference tools are applicable whether the regressors are stationary or not, and so researchers do not have to conduct any preliminary analysis of a given covariate before carrying out inference in GARCH-X models. A simulation study confirms our theoretical findings, with the distribution of standard $t$-statistics showing little sensitivity to the degree of persistence of the included covariate.

Table 1. Estimates of memory parameter $d_{x}$ and $\operatorname{AR}(1)$ coefficient for various time series

\begin{tabular}{lcccc}
\hline time series & $\hat{d}_{x}$ & AR coefficient & sample period & $T$ \\
\hline 3M treasury bill rate level & 0.94 & 1.00 & $1996 / 01 / 02-2009 / 02 / 27$ & 3434 \\
Bond yield spread (AAA-BAA) & 0.88 & 0.99 & $1987 / 11 / 02-2003 / 06 / 30$ & 3938 \\
RV of Dow Jones Industrials & 0.46 & 0.66 & $1996 / 01 / 03-2009 / 02 / 27$ & 3261 \\
RV of CAC 40 & 0.44 & 0.66 & $1996 / 01 / 03-2009 / 02 / 27$ & 3301 \\
RV of FTSE 100 & 0.42 & 0.64 & $1996 / 01 / 03-2009 / 02 / 27$ & 2844 \\
RV of German DAX & 0.42 & 0.66 & $1996 / 01 / 03-2009 / 02 / 27$ & 3296 \\
RV of British Pound & 0.56 & 0.88 & $1999 / 01 / 04-2009 / 03 / 01$ & 2576 \\
RV of Euro & 0.34 & 0.67 & $1999 / 01 / 04-2009 / 03 / 01$ & 2592 \\
RV of Swiss Franc & 0.43 & 0.69 & $1999 / 01 / 04-2009 / 03 / 01$ & 2571 \\
RV of Japanese Yen & 0.47 & 0.70 & $1999 / 01 / 04-2009 / 03 / 01$ & 2590 \\
\hline
\end{tabular}

Notes: $\hat{d}_{x}$ is the log periodogram estimate of the memory parameter $d_{x}$ and $T$ is the number of observations. RV represents the realized variance of return series. All realized variance series are from 'Oxford-Man Institute's realised library', produced by Heber et al. (2009). ${ }^{1}$ All time series are at the daily frequency.

Our theoretical results have important antecedents in the literature. Our theoretical results for the non-stationary case rely on some of the results developed in Han (2014) and Han and Park (2013) who analyze the time series properties of GARCH-X models with long-memory regressors. Kristensen and Rahbek (2005) provided theoretical results for the QMLE in the linear ARCH-X models in the case of stationary regressors. We extend their theoretical results to allow for lagged values of the volatility in the specification and non-stationary regressors. Jensen and Rahbek (2004) and Francq and Zakoïan (2012) analyzed the QMLE in the pure GARCH model (i.e., no covariates

\footnotetext{
${ }^{1}$ See http://realized.oxford-man.ox.ac.uk/.
} 
included, $\pi=0)$ and showed that the QMLE of $(\alpha, \beta)$ remained consistent and $\sqrt{n}$-asymptotically normally distributed even when $\sigma_{t}^{2}(\vartheta)$ was explosive. On the other hand, they found that $\omega$ is not identified when the volatility process is non-stationary. Our results for the QMLE of $\theta$ are similar: It remains consistent and $\sqrt{n}$-asymptotically normally distributed independently of whether $x_{t}^{2}$, and thereby $\sigma_{t}^{2}(\vartheta)$, is explosive or not. However, in contrast to the pure GARCH model, it is possible to identify and consistently estimate $\omega$ in the GARCH-X model even when $x_{t}$ is nonstationary. But the QMLE of of $\omega$ converges at a slower rate in this case. The contrasting results regarding $\omega$ are due to the fact that the dynamics of a non-stationary pure GARCH process are very different from those of a GARCH-X process with non-stationarity being induced through an exogenous long-memory process.

Finally, Han and Park (2012), henceforth HP2012, established the asymptotic theory of the QMLE for a GARCH-X model where a nonlinear transformation of a unit root process was included as exogenous regressor. Our work complements HP2012 in that we allow for a wider range of dependence in the regressor, but on the other hand do not consider general nonlinear transformations of the variable. In the special case with $d_{x}=1$, our results for the estimation of $\theta$ coincide with those of HP2012 with their transformation chosen as the quadratic function. At a technical level, we provide a more detailed analysis of the QMLE compared to HP2012. While HP2012 conjectured that $\omega$ was not identified and so kept the parameter fixed at its true value in their analysis, we here show that in fact $\omega$ can be consistently estimated from data and derive the large-sample distribution of its QMLE. This last result is derived by extending some novel limit results for non-stationary regression models developed in Wang and Phillips (2009a,b).

The rest of the paper is organized as follows. Section 2 introduces the model and the QMLE. Section 3 derives the asymptotic theory of the QMLE and their corresponding $t$-statistics for the stationary and non-stationary case. The results of a simulation study is presented in Section 4. Section 5 concludes. Proofs of theorems have been relegated to Appendix A, while proofs of lemmas can be found in the supplemental material. Before we proceed, a word on notation: Standard terminologies and notations employed in probability and measure theory are used throughout the paper. Notations for various convergences such as $\rightarrow_{\text {a.s. }}, \rightarrow_{p}$ and $\rightarrow_{d}$ frequently appear, where all limits are taken as $n \rightarrow \infty$ except where otherwise indicated.

\section{Model and Estimator}

The GARCH-X model is given by eqs. (1)-(2) where the parameters are collected in $\vartheta=(\omega, \theta)$ where $\theta=(\alpha, \beta, \pi) \in \Theta \subseteq \mathbb{R}^{3}$ and $\omega \in \mathcal{W} \subseteq[0, \infty)$. The chosen decomposition of the full parameter vector into $\theta$ and the intercept $\omega$ is due to the special role played by the latter in the non-stationary case. The true data-generating parameter is denoted $\vartheta_{0}=\left(\omega_{0}, \theta_{0}^{\prime}\right)^{\prime}$, where $\theta_{0}=\left(\alpha_{0}, \beta_{0}, \pi_{0}\right)^{\prime}$ and the associated volatility process $\sigma_{t}^{2}=\sigma_{t}^{2}\left(\vartheta_{0}\right)$. We will throughout assume that $\mathbb{E}\left[\log \left(\alpha_{0} \varepsilon_{t}^{2}+\beta_{0}\right)\right]<0$ so that non-stationarity can only be induced by $x_{t}$. In particular, if $x_{t}$ is stationary then $\sigma_{t}^{2}$ and $y_{t}$ are stationary; see Section 3.1 for details. In the stationary case, we impose no further restrictions on its time series dynamics. On the other hand, in the non-stationary case, we restrict $x_{t}$ to be a 
long-memory process of the form

$$
x_{t}=x_{t-1}+\xi_{t}
$$

where, for a sequence $\left\{v_{t}\right\}$ which is i.i.d. $\left(0, \sigma_{v}^{2}\right)$,

$$
(1-L)^{d} \xi_{t}=v_{t}, \quad-1 / 2<d<1 / 2
$$

Hence, $x_{t}$ is an $I\left(d_{x}\right)$ process with $d_{x}=d+1 \in(1 / 2,3 / 2)$. Note that $\left\{\varepsilon_{t}\right\}$ and $\left\{v_{t}\right\}$ are allowed to be dependent. Hence, the model can accommodate leverage effects catered for by the GJR-GARCH model if $\left\{\varepsilon_{t}\right\}$ and $\left\{v_{t}\right\}$ are negatively correlated. See Han (2014) for more details on the model and its time series properties.

Dittmann and Granger (2002) analyzed the properties of $x_{t}^{2}$ given $x_{t}$ is fractionally integrated and showed that, when $x_{t}$ is a Gaussian fractionally integrated process of order $d_{x}$, then $x_{t}^{2}$ is asymptotically also a long memory process of order $d_{x^{2}}=d_{x}$. Hence, for $1 / 2<d_{x}<3 / 2$, the covariate $x_{t}^{2}$ is non-stationary long memory, including the case of unit root-type behaviour. Considering that the range of memory parameter for real data used as covariates in the literature seldom exceeds unity, the range of $d_{x}$ we consider is wide enough to cover all covariates used in the empirical literature.

Whether $x_{t}$ is stationary or not, we will require it to be exogeneous in the sense that $\mathbb{E}\left[\varepsilon_{t} \mid x_{t-1}\right]=$ 0 and $\mathbb{E}\left[\varepsilon_{t}^{2} \mid x_{t-1}\right]=1$. This restricts the choices of $x_{t}$; for example, in most situations, the exogeneity assumption will be violated if $y_{t}$ is a stock return, say, $r_{1, t}$ and $x_{t-1}=r_{2, t}$ is another return series since these will in general be contemporaneously correlated. This in turn will generate simultaneity biases in the estimation of the GARCH-X model similar to OLS in simultaneous equations models. If instead $x_{t-1}=r_{2, t-1}$, the GARCH-X model can be thought of as a restricted version of a bivariate GARCH model where lags of $r_{1, t}$ do not affect the volatility of $r_{2, t}$ and only the first lag of $r_{2, t}$ affects the volatility of $r_{1, t}$. This restriction may in some cases be implausible. On the other hand, GARCH-X models is a lot simpler to estimate compared to a bivariate GARCH model: The former only contains four parameters while a bivariate BEKK-GARCH $(1,1)$ contains twelve parameters.

Our model is related to the one considered in HP2012 given by $\sigma_{t}^{2}(\vartheta)=\alpha y_{t-1}^{2}+\beta \sigma_{t-1}^{2}(\vartheta)+$ $f\left(x_{t-1}, \gamma\right)$, where $x_{t}$ is integrated or near-integrated, and $f\left(x_{t-1}, \gamma\right)$ is a positive, asymptotically homogeneous function as introduced by Park and Phillips (1999). ${ }^{2}$ If we let $d_{x}=1$ in our model, $x_{t}$ is integrated and our model belongs to the model considered by HP2012 with $f\left(x_{t-1}, \gamma\right)=\omega+\pi x_{t-1}^{2}$. While their model allows for more general nonlinear transformations of $x_{t}$, our analysis includes more general dependence structure of $x_{t}$ : It is either stationarity or it is fractionally integrated process with $1 / 2<d_{x}<3 / 2$. As shown in Table 1 , these are empirically relevant types of dynamic behavior.

Let $\left(y_{t}, x_{t-1}\right)$ for $t=0, \ldots, n$, be $n+1 \geq 2$ observations from (1)-(2). We then consider estimation

\footnotetext{
${ }^{2}$ Note a notational difference in HP2012: Instead of $f\left(x_{t-1}, \gamma\right)$, HP2012 use $f\left(x_{t}, \gamma\right)$ where $x_{t}$ is adapted to $\mathcal{F}_{t-1}$
} 
of $\vartheta_{0}$ using the Gaussian $\log$-likelihood with $\varepsilon_{t} \sim$ i.i.d. $N(0,1)$ :

$$
L_{n}(\vartheta)=\sum_{t=1}^{n} \ell_{t}(\vartheta), \quad \ell_{t}(\vartheta)=-\log \sigma_{t}^{2}(\vartheta)-\frac{y_{t}^{2}}{\sigma_{t}^{2}(\vartheta)}
$$

where $\sigma_{t}^{2}(\vartheta)$ is given in eq. (2). The volatility process is assumed to be initialized at some fixed parameter independent value $\bar{\sigma}_{0}^{2}>0, \sigma_{0}^{2}(\vartheta)=\bar{\sigma}_{0}^{2}$. We will not restrict $\varepsilon_{t}$ to be normally distributed and hence $L_{n}(\vartheta)$ is a quasi-log likelihood. The QMLE of $\vartheta_{0}$ is then defined as:

$$
\hat{\vartheta}=(\hat{\omega}, \hat{\theta})=\arg \max _{(\omega, \theta) \in \mathcal{W} \times \Theta} L_{n}(\omega, \theta)
$$

\section{Asymptotic Theory}

The main arguments used to establish the asymptotic distribution of the QMLE are identical for the two cases - stationary or non-stationary regressors. The technical tools used to establish the main arguments differ in the two cases though, and so we provide separate proofs for them. But first, we outline the proof strategy for consistency and asymptotic normality of the QMLE to emphasise similarities and differences in the analysis of the two different cases.

To present the arguments in a streamlined fashion, it proves useful to redefine $\ell_{t}(\vartheta)$ as a normalized version of the log-likelihood function by subtracting the log-likelihood evaluated at $\vartheta_{0}$,

$$
\ell_{t}(\vartheta):=\left\{-\log \sigma_{t}^{2}(\vartheta)-\frac{y_{t}^{2}}{\sigma_{t}^{2}(\vartheta)}\right\}-\left\{-\log \sigma_{t}^{2}-\frac{y_{t}^{2}}{\sigma_{t}^{2}}\right\}=-\log \left(r_{t}(\vartheta)\right)-\left\{\frac{1}{r_{t}(\vartheta)}-1\right\} \varepsilon_{t}^{2}
$$

where $\sigma_{t}^{2}$ denotes the true data-generating volatility process,

$$
\sigma_{t}^{2}=\omega_{0}+\alpha_{0} y_{t-1}^{2}+\beta_{0} \sigma_{t-1}^{2}+\pi_{0} x_{t-1}^{2},
$$

and $r_{t}(\vartheta)$ is a variance-ratio process defined as

$$
r_{t}(\vartheta):=\frac{\sigma_{t}^{2}(\vartheta)}{\sigma_{t}^{2}}
$$

This normalization does not affect the QMLE since $-\log \sigma_{t}^{2}-y_{t}^{2} / \sigma_{t}^{2}$ is parameter independent.

Note that the process $r_{t}(\vartheta)$ is in general not stationary since $\sigma_{t}^{2}(\vartheta)$ has been initialized at some fixed value and $x_{t}$ may be non-stationary. For consistency, the main argument involves showing that the normalized version of the log-likelihood satisfies

$$
\sup _{\vartheta \in \mathcal{W} \times \Theta} \frac{1}{n}\left\|L_{n}(\vartheta)-L_{n}^{*}(\vartheta)\right\| \rightarrow^{P} 0
$$


where $L_{n}^{*}(\vartheta)$ is given by

$$
L_{n}^{*}(\vartheta)=\sum_{t=1}^{n} \ell_{t}^{*}(\vartheta), \quad \ell_{t}^{*}(\vartheta)=-\log \left(r_{t}^{*}(\vartheta)\right)-\left\{\frac{1}{r_{t}^{*}(\vartheta)}-1\right\} \varepsilon_{t}^{2},
$$

and $r_{t}^{*}(\vartheta)$ is a stationary sequence which is asymptotically equivalent to $r_{t}(\vartheta)$. We can now appeal to a uniform Law of Large Numbers (LLN) for stationary and ergodic sequences to obtain that $L_{n}^{*}(\vartheta) / n \rightarrow p L^{*}(\vartheta):=\mathbb{E}\left[\ell_{t}^{*}(\vartheta)\right]$ uniformly in $\vartheta$. The precise definition of $r_{t}^{*}(\vartheta)$, and thereby $L^{*}(\vartheta)$, depends on whether $x_{t}$ is stationary or not. In particular, in the stationary case $\vartheta_{0}=$ $\arg \max _{\vartheta} L^{*}(\vartheta)$ is uniquely identified and so $\hat{\vartheta} \rightarrow p \vartheta_{0}$ globally, while in the nonstationary case $L^{*}(\vartheta)=L^{*}(\theta)$ is constant w.r.t. $\omega$ and so we can only conclude that $\hat{\theta} \rightarrow_{p} \theta_{0}$. This would seem to indicate that in the non-stationary case $\hat{\omega}$ is inconsistent which would be similar to the explosive pure GARCH model as analyzed by Jensen and Rahbek (2004) and Francq and Zakoïan (2012). However, in our case, this conclusion is not correct and is an artifact of normalizing $L_{n}(\vartheta)$ by $1 / n$. By analyzing the local behaviour of $L_{n}(\vartheta)$ in a shrinking neighbourhood of $\vartheta_{0}$, we find that in the non-stationary case $\hat{\omega}$ remains consistent but converges at a slower rate compared to $\hat{\theta}$.

To derive the asymptotic distribution of $\hat{\vartheta}$, we proceed to analyze the score and hessian of the quasi-log likelihood. We denote the score vector by $S_{n}(\vartheta)=\left(S_{n, \omega}(\vartheta), S_{n, \theta}(\vartheta)^{\prime}\right)^{\prime} \in \mathbb{R}^{4}$, where $S_{n, \omega}(\vartheta)=\partial L_{n}(\vartheta) /(\partial \omega) \in \mathbb{R}$ and $S_{n, \theta}(\vartheta)=\partial L_{n}(\vartheta) /(\partial \theta) \in \mathbb{R}^{3}$ and the Hessian matrix by

$$
H_{n}(\vartheta)=\left[\begin{array}{cc}
H_{n, \omega \omega}(\vartheta) & H_{n, \omega \theta}(\vartheta) \\
H_{n, \theta \omega}(\vartheta) & H_{n, \theta \theta}(\vartheta)
\end{array}\right] \in \mathbb{R}^{4 \times 4}
$$

where $H_{n, \theta \omega}(\vartheta)=\partial^{2} L_{n}(\vartheta) /(\partial \theta \partial \omega) \in \mathbb{R}^{3}$ and the other components are defined similarly. A standard first order Taylor expansion of the score vector yields $0=S_{n}(\hat{\vartheta})=S_{n}\left(\vartheta_{0}\right)+H_{n}(\bar{\vartheta})\left(\hat{\vartheta}-\vartheta_{0}\right)$, where $\bar{\vartheta}$ lies on the line segment connecting $\hat{\vartheta}$ and $\vartheta_{0}$. Assuming that $\vartheta_{0}$ lies in the interior of the parameter space, $\hat{\vartheta}$ must be an interior solution with probability approaching one (w.p.a.1). That is, $S_{n}(\hat{\vartheta})=0$ w.p.a.1. What remains is to derive the limiting distribution of $S_{n}\left(\vartheta_{0}\right)$ and $H_{n}(\bar{\vartheta})$.

In the stationary case, we can appeal to LLN and Central Limit Theorem (CLT) for stationary and ergodic sequences to show that

$$
S_{n}\left(\vartheta_{0}\right) / \sqrt{n} \rightarrow_{d} N\left(0, \Sigma^{\mathrm{st}}\right), \quad-H_{n}(\bar{\vartheta}) / n \rightarrow{ }_{p} H^{\mathrm{st}}>0,
$$

where $\Sigma^{\text {st }} \in \mathbb{R}^{4 \times 4}$ are $H^{\text {st }} \in \mathbb{R}^{4 \times 4}$ are constant. This implies that

$$
\sqrt{n}\left(\hat{\vartheta}-\vartheta_{0}\right) \rightarrow{ }_{d} N\left(0, \Omega^{\mathrm{st}}\right), \quad \Omega^{\mathrm{st}}=\left(H^{\mathrm{st}}\right)^{-1} \Sigma^{\mathrm{st}}\left(H^{\mathrm{st}}\right)^{-1}
$$

In the non-stationary case, the score and hessian, and thereby the QMLE's, have different asymptotic behaviour. First of all, $\hat{\omega}$ and $\hat{\theta}$ converge at different rates which we collect in the matrix $V_{n}$,

$$
V_{n}:=\left[\begin{array}{cc}
n^{1 / 4-d / 2} & O_{1 \times 3} \\
O_{3 \times 1} & n^{1 / 2} I_{3}
\end{array}\right] \in \mathbb{R}^{4 \times 4}
$$


where $O_{k \times m} \in \mathbb{R}^{k \times m}$ denotes the matrix of zeros and $I_{k} \in \mathbb{R}^{k \times k}$ denotes the identity matrix. We then show that

$$
V_{n}^{-1} S_{n}\left(\vartheta_{0}\right) \rightarrow_{d} M N\left(0, \Sigma^{\mathrm{nst}}\right), \quad-V_{n}^{-1} H_{n}(\bar{\vartheta}) V_{n}^{-1} \rightarrow_{d} H^{\mathrm{nst}}>0,
$$

where $M N\left(0, \Sigma^{\text {nst }}\right)$ denotes a mixed-normal distribution with (random) covariance matrix $\Sigma^{\text {nst }} \in$ $\mathbb{R}^{4 \times 4}$, and $H^{\text {nst }} \in \mathbb{R}^{4 \times 4}$ is also random. The proof of eq. (14) employs generalized versions of limit results for fractionally integrated processes developed in Wang and Phillips (2009a) that we have collected in Lemma 6 below. Having established (14), it follows by standard arguments that

$$
V_{n}\left(\hat{\vartheta}-\vartheta_{0}\right) \rightarrow_{d} M N\left(0, \Omega^{\mathrm{nst}}\right), \quad \Omega^{\mathrm{nst}}=\left(H^{\mathrm{nst}}\right)^{-1} \Sigma^{\mathrm{nst}}\left(H^{\mathrm{nst}}\right)^{-1}
$$

In particular, $\hat{\theta}$ is $\sqrt{n}$-asymptotically normally distributed while $\hat{\omega}$ converges with a slower rate of $n^{1 / 4-d / 2}$ and follows a mixed-normal distribution. Importantly, in comparison to pure explosive GARCH models where $\omega_{0}$ is not identified, we can still conduct inference about $\omega_{0}$ when the explosiveness is induced by a long-memory regressor.

In conclusion, the asymptotic distribution of $\hat{\vartheta}$ depends on whether $x_{t}$ is stationary or not. Fortunately, the distribution is in both cases mixed-normal and so standard test statistics prove to be robust to the degree of persistence of $x_{t}$. In particular, we show that standard $t$-statistics follow $N(0,1)$ distributions irrespective of the regressor's level of persistence. The reason for this result is that in the computation of the t-statistics, we pre-multiply the QMLE's with an estimator of its large-sample co-variance matrix. This normalization takes out the random covariance matrix, $\Omega^{\text {nst }}$, that appears in the limiting distribution in the non-stationary case.

Since the assumptions and techniques used to establish the above results differ depending on whether $x_{t}$ is stationary or not, we consider the two cases in turn: The following subsection covers the stationary case, while the subsequent one focuses on the non-stationary case. Based on these results, the asymptotic properties of the $t$-statistics are analyzed in Section 3.3.

\subsection{QMLE in Stationary Case}

We first show that the QMLE is globally consistent under the following conditions with $\mathcal{F}_{t}$ denoting the natural filtration:

\section{Assumption 1}

(i) $\left\{\left(\varepsilon_{t}, x_{t}\right)\right\}$ is stationary and ergodic with $\mathbb{E}\left[\varepsilon_{t} \mid \mathcal{F}_{t-1}\right]=0$ and $\mathbb{E}\left[\varepsilon_{t}^{2} \mid \mathcal{F}_{t-1}\right]=1$.

(ii) $\mathbb{E}\left[\log \left(\alpha_{0} \varepsilon_{t}^{2}+\beta_{0}\right)\right]<0$ and $\mathbb{E}\left[x_{t}^{2 q}\right]<\infty$ for some $0<q<\infty$.

(iii) $\Theta=\{\vartheta: \underline{\omega} \leq \omega \leq \bar{\omega}, 0 \leq \alpha \leq \bar{\alpha}, 0 \leq \beta \leq \bar{\beta}, 0 \leq \pi \leq \bar{\pi}\}$, where $0<\underline{\omega} \leq \bar{\omega}<\infty, \bar{\alpha}<\infty$, $\bar{\beta}<1$ and $\bar{\pi}<\infty$. The true value $\vartheta_{0} \in \Theta$ with $\left(\alpha_{0}, \pi_{0}\right) \neq(0,0)$.

(iv) For any $(a, b) \neq(0,0): a \varepsilon_{t}^{2}+b x_{t}^{2} \mid \mathcal{F}_{t-1}$ has a nondegenerate distribution. 
Assumption 1(i) is a generalization of the conditions found in Escanciano (2009) who derives the asymptotic properties of QMLE for pure GARCH processes (that is, no exogenous covariates are included) with martingale difference errors. The assumption is weaker than the i.i.d. assumption imposed in Kristensen and Rahbek (2005). The moment conditions in Assumption 1(ii) implies that a stationary solution to eqs. (1)-(2) at the true parameter value $\vartheta_{0}$ exists and has a finite polynomial moment, c.f. Lemma 1 below. We here allow for integrated GARCH processes $(\alpha+\beta=1)$, and impose very weak moment restrictions on the regressor. We do however rule out explosive volatility when $x_{t}$ is stationary; we expect that the arguments of Jensen and Rahbek (2004) can be extended to GARCH-X models with $\mathbb{E}\left[\log \left(\alpha_{0} \varepsilon_{t-1}^{2}+\beta_{0}\right)\right]>0$, thereby showing that $\hat{\theta}$ is consistent while $\hat{\omega}$ is inconsistent. The compactness condition in Assumption 1(iii) should be possible to weaken by following the arguments of Kristensen and Rahbek (2005); this will lead to more complicated proofs though and so we maintain the compactness assumption here for simplicity. The requirement that $\left(\alpha_{0}, \pi_{0}\right) \neq(0,0)$ is needed to ensure identification of $\beta_{0}$ since in the case where $\left(\alpha_{0}, \pi_{0}\right)=(0,0)$, $\sigma_{t}^{2}=\sigma_{t}^{2}\left(\vartheta_{0}\right) \rightarrow a . s . \omega_{0} /\left(1-\beta_{0}\right)$ and so we would not be able to jointly identify $\omega_{0}$ and $\beta_{0}$. The nondegeneracy condition in Assumption 1(iv) is also needed for identification. It rules out (dynamic) collinearity between $y_{t-1}^{2}$ and $x_{t}^{2}$. It is similar to the no-collinearity restriction imposed in Kristensen and Rahbek (2005).

To derive the asymptotic properties of $\hat{\vartheta}$, we establish some preliminary results. The first lemma states that a stationary solution to the model at the true parameter values exists:

Lemma 1 Under Assumption 1: There exists a stationary and ergodic solution to eqs. (1)-(2) at $\vartheta_{0}$ satisfying $\mathbb{E}\left[\sigma_{t}^{2 s}\right]<\infty$ and $\mathbb{E}\left[y_{t}^{2 s}\right]<\infty$ for some $0<s<1$.

We will in the following work under the implicit assumption that we have observed the stationary solution. Next, we show that for any value of $\vartheta$ in the parameter space, the volatility-ratio process $r_{t}(\vartheta)$ is well-approximated by a stationary version:

Lemma 2 Under Assumption 1: With $s>0$ given in Lemma 1, there exists some $K_{s}<\infty$ such that

$$
\mathbb{E}\left[\sup _{\vartheta \in \mathcal{W} \times \Theta}\left|r_{t}(\vartheta)-r_{t}^{*}(\vartheta)\right|^{s}\right] \leq K_{s} \beta^{s t}
$$

where

$$
r_{t}^{*}(\vartheta):=\frac{\sigma_{0, t}^{2}(\vartheta)}{\sigma_{t}^{2}}, \quad \sigma_{0, t}^{2}(\vartheta):=\sum_{i=1}^{\infty} \beta^{i-1}\left(\omega+\alpha y_{t-i}^{2}+\pi x_{t-i}^{2}\right) .
$$

The process $\sigma_{0, t}^{2}(\vartheta)$ is stationary and ergodic with $\mathbb{E}\left[\sup _{\vartheta \in \mathcal{W} \times \Theta} \sigma_{0, t}^{2 s}(\vartheta)\right]<\infty$.

Note that, in particular, $\sigma_{t}^{2}=\sigma_{0, t}^{2}\left(\vartheta_{0}\right)$. This in turn implies that eq. (8) holds with $r_{t}^{*}(\vartheta)$ defined in the previous lemma. With these results in hand, we are now ready to show the first main result of this section:

Theorem 3 Under Assumption 1, the QMLE $\hat{\vartheta}$ is consistent. 
Having shown that the QMLE is consistent, we proceed to verify eq. (11) under the following additional assumption:

\section{Assumption 2}

(i) $\kappa_{4}=\mathbb{E}\left[\left(\varepsilon_{t}^{2}-1\right)^{2} \mid \mathcal{F}_{t-1}\right]<\infty$ is constant.

(ii) $\vartheta_{0}$ is in the interior of $\Theta$.

Assumption 2(i) is used to show that the variance of the score exists. It could be weakened to allow for $\mathbb{E}\left[\left(\varepsilon_{t}^{2}-1\right)^{2} \mid \mathcal{F}_{t-1}\right]$ to be time-varying as in Escanciano (2009), but for simplicity and to allow for easier comparison with the results in the non-stationary case, we maintain Assumption 2(i). Assumption 2(ii) is needed in order to ensure that $S_{n}(\hat{\vartheta})=0$ w.p.a.1.

As a first step towards eq. (11), the following lemma proves useful. It basically shows that the derivatives of the volatility-ratio process $r_{t}^{*}(\vartheta)$ are stationary with suitable moments:

Lemma 4 Under Assumptions 1-2: $\partial r_{t}^{*}(\vartheta) /(\partial \vartheta)$ and $\partial^{2} r_{t}^{*}(\vartheta) /\left(\partial \vartheta \partial \vartheta^{\prime}\right)$ are stationary and ergodic for all $\vartheta \in \mathcal{W} \times \Theta$. Moreover, there exists stationary and ergodic sequences $B_{k, t} \in \mathcal{F}_{t-1}$, $k=0,1,2$, which are independent of $\vartheta$ such that

$$
\frac{1}{r_{t}^{*}(\vartheta)} \leq B_{0, t}, \quad \frac{\left\|\partial r_{t}^{*}(\vartheta) /(\partial \vartheta)\right\|}{r_{t}^{*}(\vartheta)} \leq B_{1, t}, \quad \frac{\left\|\partial^{2} r_{t}^{*}(\vartheta) /\left(\partial \vartheta \partial \vartheta^{\prime}\right)\right\|}{r_{t}^{*}(\vartheta)} \leq B_{2, t}
$$

for all $\vartheta$ in a neighbourhood of $\vartheta_{0}$, where $\mathbb{E}\left[B_{1, t}+B_{2, t}^{2}\right]<\infty$ and $\mathbb{E}\left[B_{0, t}\left\{B_{1, t}+B_{2, t}^{2}\right\}\right]<\infty$.

This lemma is used to construct suitable bounds for the score and hessian that allow us to appeal to CLT and LLN for stationary and ergodic sequences, and thereby establishing eq. (11):

Theorem 5 Under Assumptions 1-2, the QMLE $\hat{\vartheta}$ satisfies eq. (12) where, with $\kappa_{4}$ given in Assumption 2 and $r_{t}^{*}(\vartheta)$ in eq. (16), $\Sigma^{s t}=\kappa_{4} H^{s t}$ and $H^{s t}=\mathbb{E}\left[\frac{\partial r_{t}^{*}\left(\vartheta_{0}\right)}{\partial \vartheta} \frac{\partial r_{t}^{*}\left(\vartheta_{0}\right)}{\partial \vartheta^{\prime}}\right]$.

\subsection{QMLE in Non-stationary Case}

For consistency, we follow a similar strategy to develop the asymptotic properties of the QMLE when $x_{t}^{2}$ is explosive, except a different variance-ratio approximation has to be used. To develop this variance-ratio approximation, we utilize some results derived in Han and Park (2013). We impose the following conditions on the model which are stronger than the ones imposed in the stationary case, but on the other hand allow for non-stationary regressors:

\section{Assumption 3}

(i) $\left\{\varepsilon_{t}\right\}$ and $\left\{v_{t}\right\}$ are i.i.d., mutually independent, and satisfies $\mathbb{E}\left[\varepsilon_{t}\right]=\mathbb{E}\left[v_{t}\right]=0, \mathbb{E}\left[\varepsilon_{t}^{2}\right]=1$, and $\mathbb{E}\left[\left|v_{t}\right|^{p}\right]<\infty$ for some $p \geq 2$. 
(ii) $\Theta=\left\{\theta \in \mathbb{R}^{3}: \underline{\alpha} \leq \alpha \leq \bar{\alpha}, \underline{\beta} \leq \beta \leq \bar{\beta}, \underline{\pi} \leq \pi \leq \bar{\pi}\right\}$ and $\mathcal{W}=[\underline{\omega}, \bar{\omega}]$ where $0<\underline{\alpha}<\bar{\alpha}<\infty$, $0<\underline{\beta}<\bar{\beta}<1,0<\underline{\pi}<\bar{\pi}<\infty$ and $0<\underline{\omega}<\bar{\omega}<\infty$.

(iii) $\left\{x_{t}\right\}$ solves eqs. (3)-(4) with $d \in(-1 / 2,1 / 2)$.

(iv) $\mathbb{E}\left[\left|\varepsilon_{t}\right|^{q}\right]<\infty$ and $\mathbb{E}\left[\left(\beta_{0}+\alpha_{0} \varepsilon_{t}^{2}\right)^{q / 2}\right]<1$ for some $q>4$.

(v) $1 / p+2 / q<1 / 2+d$.

Assumption 3(i) requires the errors driving the model to be i.i.d. which is stronger than Assumption 1(i). We expect that it could be weakened to allow for some dependence, but this would greatly complicate the analysis. Similarly, the mutual independence of $\left\{\varepsilon_{t}\right\}$ and $\left\{v_{t}\right\}$ is a technical assumption and only used to establish the LLN and CLT in Lemma 6. Since Lemma 6 is only used in the analysis of $\hat{\omega}$, the proof of consistency and asymptotic normality of $\hat{\theta}$ is valid without the independence assumption. We conjecture that Lemma 6 , and thereby the asymptotic properties of $\hat{\omega}$ as stated below, holds under weaker assumptions than independence, but this requires a different proof technique; see Wang (2013). Assumption 3(ii) restricts the parameters to be strictly positive; this is used when showing that $r_{t}(\vartheta)$ is well-approximated by a stationary version uniformly over $\vartheta$. A similar restriction is found in Francq and Zakoïan (2012). Assumption 3(iii) precisely defines the covariate $\left\{x_{t}\right\}$ as an $I\left(d_{x}\right)$ process with $1 / 2<d_{x}<3 / 2$. This restriction on $d_{x}$ is imposed in order to employ the results of Han and Park (2013) and the limit results in Lemma 6 below.

Assumptions 3(iv)-(v) correspond to Assumptions 2(b)-(c) in HP2012. Assumption 3(iv) introduces some moments conditions for the innovation sequences $\left\{v_{t}\right\}$ and $\left\{\varepsilon_{t}\right\}$. It is stronger than $\mathbb{E}\left[\log \left(\beta+\alpha \varepsilon_{t}^{2}\right)\right]<0$ as imposed in Assumption 1(ii). In particular, while $\alpha+\beta=1$ is allowed for the stationary case in the previous section, (iv) rules this out in the nonstationary case. We do not find this restrictive though since, when $x_{t}$ is an $\mathrm{I}(1)$ process and $\alpha+\beta=1$, $y_{t}^{2}$ has $\mathrm{I}(2)$ type behaviour which is not very likely for most economic and financial time series. Moreover, in most applications, when additional regressors are included, it is usually found that $\alpha+\beta<1$ so this restriction does not appear restrictive from an empirical point of view. Together Assumptions 3 (iv)-(v) can lead to quite strong moment restrictions. For example, if $d$ is close to $-1 / 2$, then $p$ and $q$ have to be chosen very large for the inequality in Assumption 3(v) to hold. These are used when developing the stationary approximation of the volatility ratio process $r_{t}(\vartheta)$ which relies on the existence of certain moments. We conjecture that our theory would go through under weaker moment restrictions, but unfortunately we have not been able to demonstrate this here.

For the proof of the non-stationary case, we first present some additional notation and useful results. Let $D[0,1]$ be the space of cadlag functions on $[0,1]$ equipped with the uniform metric, and $\Rightarrow$ denote weak convergence on $D[0,1]$. Also, let $L_{W_{d}}(t, x)$ denote the local time of a fractional Brownian motion and $K>0$ a normalizing constant (see Wang and Phillips, 2009a for precise definitions). Then the following theorem, which proves fundamental in establishing the necessary limit results for the score and hessian, holds:

Theorem 6 Let $\left\{x_{t}\right\}$ satisfy Assumption 3(iii) and $f(x)$ be an integrable function. 
(i) Suppose $\left\{w_{t}\right\}$ is stationary, independent of $\left\{x_{t}\right\}$, and satisfies $\sum_{t=1}^{\infty}\left|\operatorname{Cov}\left(w_{0}, w_{t}\right)\right|<\infty$. Then,

$$
\frac{1}{n^{1 / 2-d}} \sum_{t=1}^{[n s]} f\left(x_{t-1}\right) w_{t} \Rightarrow L_{W_{d}}(s, 0) \times K \mathbb{E}\left[w_{t}\right] \int_{-\infty}^{\infty} f(x) d x \text { on } D[0,1]
$$

(ii) Suppose in addition that $u_{t}$ is a martingale difference sequence w.r.t. a filtration $\mathcal{F}_{t}$ that $\left(x_{t-1}, w_{t}\right)$ is adapted to; $\left\{x_{t}\right\}$ and $\left\{u_{t}\right\}$ are independent, $\mathbb{E}\left[u_{t}^{2} \mid \mathcal{F}_{t-1}\right]=\sigma_{u}^{2}>0$ and $\sup _{t \geq 1} \mathbb{E}\left[\left|u_{t} w_{t}\right|^{q_{u}}\right]<$ $\infty$ a.s. for some $q_{u}>2 ; \sum_{t=1}^{\infty}\left|\operatorname{Cov}\left(w_{0}^{2}, w_{t}^{2}\right)\right|<\infty$. Then,

$$
\frac{1}{n^{1 / 4-d / 2}} \sum_{t=1}^{[n s]} f\left(x_{t-1}\right) w_{t} u_{t} \Rightarrow \sqrt{L_{W_{d}}(s, 0)} G(s)
$$

where $G(s)$ is a Gaussian process which is independent of $L_{W_{d}}(s, 0)$ and with covariance kernel $\left(s_{1} \wedge s_{2}\right) K \mathbb{E}\left[w_{t}^{2}\right] \sigma_{u}^{2} \int_{-\infty}^{\infty} f^{2}(x) d x$.

Remark 7 A sufficient condition for the assumptions on $\left\{w_{t}\right\}$ in (i) and (ii) to hold is that it is stationary and $\beta$-mixing such that, for some $\delta>0, \mathbb{E}\left[\left|w_{t}\right|^{2(1+\delta)}\right]<\infty$ and its mixing coefficients satisfy $\sum_{t=1}^{\infty} \beta_{t}^{\delta /(1+\delta)}<\infty$; see, for example, Yoshihara (1976, Lemma 1).

The above lemma is a generalization of the LLN and CLT established in Wang and Phillips (2009a) to allow for inclusion of a stationary component, $w_{t}$. It is the fundamental tool in our analysis of the score and hessian w.r.t. $\omega$ since the first and second derivative of $r_{t}(\vartheta)$ w.r.t. $\omega$ can be written on the form $f\left(x_{t-1}\right) w_{t}$ for a suitable choice of $f$ and $w_{t}$. Employing results in Han and Park (2013), we also develop a stationary approximation of the variance ratio $r_{t}(\vartheta)=\sigma_{t}^{2}(\vartheta) / \sigma_{t}^{2}$ that is used in the asymptotic analysis of the score and hessian w.r.t. $\theta$.

Lemma 8 Under Assumption 3,

$$
\sup _{\vartheta \in \mathcal{W} \times \Theta} \max _{1 \leq t \leq n}\left|r_{t}(\vartheta)-r_{t}^{*}(\theta)\right|=o_{p}(1)
$$

where, with $z_{t}=z_{t}\left(\theta_{0}\right)$,

$$
r_{t}^{*}(\theta):=\frac{z_{t}(\theta)}{z_{t}}, \quad z_{t}(\theta)=\alpha \sum_{i=1}^{\infty} \beta^{i-1} z_{t-i} \varepsilon_{t-i}^{2}+\frac{\pi}{\pi_{0}} \frac{1}{1-\beta}
$$

The sequence $r_{t}^{*}(\theta)$ is stationary and ergodic with $\mathbb{E}\left[\sup _{\theta} r_{t}^{*}(\theta)^{-k}\right]<\infty$ for any $k \in \mathbb{R}$. Moreover, $\sup _{\vartheta}\left\{\sigma_{t}^{2}\left(\vartheta_{0}\right) \sigma_{t}^{-2}(\vartheta)\right\} \leq W_{t}$, where $W_{t}$ is stationary and ergodic with $\mathbb{E}\left[W_{t}^{k}\right]<\infty$ for any $k>0$.

Lemma 8 is used to establish eq. (8). It is important to note that $r_{t}^{*}(\theta)$ does not depend on the regressor $x_{t}$ (and so is stationary), but still contains information about its regression coefficient, $\pi$. On the other hand, $r_{t}^{*}(\theta)$, and thereby $L_{n}^{*}(\vartheta)=L_{n}^{*}(\theta)$, is independent of $\omega$ and so asymptotically the log-likelihood, when normalized by $1 / n$, contains no information about this parameter in large 
samples. We are therefore only able to show global consistency of $\hat{\theta}$. However, a local analysis of $L_{n}(\vartheta)$, where Lemma 6 is used to verify the high-level conditions in Kristensen and Rahbek (2010, Lemma 11), shows that $\hat{\omega}$ is locally consistent but converges at a slower than standard rate:

Theorem 9 Under Assumption 3, $\hat{\theta} \rightarrow_{p} \theta_{0}$. Moreover, for some $\epsilon>0$, there exists a unique maximum point $\hat{\vartheta}=(\hat{\omega}, \hat{\theta})$ of $L_{n}(\vartheta)$ in $\left\{\vartheta:\left|\omega-\omega_{0}\right| \leq \epsilon, \quad n^{1 / 4+d / 2}\left\|\theta-\theta_{0}\right\| \leq \epsilon\right\}$ w.p.a.1 that satisfies $\hat{\omega}=\omega_{0}+o_{p}(1)$ and $\hat{\theta}=\theta_{0}+o_{p}\left(1 / n^{1 / 4+d / 2}\right)$.

The consistency result for $\hat{\theta}$ is a global statement where the estimator is part of the maximizer $\hat{\vartheta}$ of $L_{n}(\vartheta)$ over the whole parameter space $\mathcal{W} \times \Theta$. The second result that establishes consistency of $\hat{\omega}$ and convergence rate of $\hat{\theta}$ is a local statement with $\hat{\vartheta}$ now being a local maximizer of $L_{n}(\vartheta)$ over a shrinking set. To avoid additional notation, we here use $\hat{\theta}$ to denote both the global and local estimator. In finite samples, these two could differ if the likelihood function has a local maximum in a neighbourhood of $\theta_{0}$. Ideally, we would have carried out a global analysis of $\hat{\omega}$ as well and established consistency of it on $\mathcal{W}$. However, to our knowledge, there exists no results for global consistency for non-linear estimators whose components converge at different rates; see e.g. Kristensen and Rahbek (2010).

Next, we analyze the asymptotic distribution of $\vartheta$ by applying the general result of Kristensen and Rahbek (2010, Lemma 12) to our setting:

Theorem 10 Let Assumption 3 hold. Then eq. (15) holds with $\Sigma^{n s t}=\kappa_{4} H^{n s t}$ and

$$
H^{n s t}=\left[\begin{array}{cc}
H_{\omega \omega}^{n s t} & O_{1 \times 3} \\
O_{3 \times 1} & H_{\theta \theta}^{n s t}
\end{array}\right] \in \mathbb{R}^{4 \times 4} .
$$

where

$$
\begin{aligned}
H_{\omega \omega}^{n s t} & =K \frac{\mathbb{E}\left[1 / z_{t}^{2}\right]}{\left(1-\beta_{0}\right)^{2}} \int_{-\infty}^{\infty}\left(\frac{1}{\omega_{0}+\pi_{0} s^{2}}\right)^{2} d s \times L_{W_{d}}(1,0), \\
H_{\theta \theta}^{n s t} & =\mathbb{E}\left[\frac{\partial r_{t}^{*}\left(\theta_{0}\right)}{\partial \theta} \frac{\partial r_{t}^{*}\left(\theta_{0}\right)}{\partial \theta^{\prime}}\right] \in \mathbb{R}^{3 \times 3} .
\end{aligned}
$$

Note here that the estimators $\hat{\theta}$ and $\hat{\omega}$ are asymptotically independent and that the limiting covariance matrix $H_{\theta \theta}^{\text {nst }}$ for the QMLE of $\theta$ is non-random. Thus, it is only the limiting distribution of $\hat{\omega}$ which is mixed-normal since $H_{\omega \omega}^{\text {nst }}$ is random.

\subsection{Robust Inference}

Comparing Theorems 5 and 10, we see that the large-sample distribution of the QMLE changes quite substantially when we move from the stationary case to the non-stationary one. One could therefore fear that, for a chosen regressor, inference would be dependent on whether $x_{t}$ is stationary or not. However, in both cases, the limiting distribution of the QMLE is mixed normal with the 
(possibly random) covariance matrix being the product of limits of the (appropriately scaled) score and hessian. Whether $x_{t}$ is stationary or not, a natural estimator of the covariance matrix is

$$
\hat{\Omega}=H_{n}^{-1}(\hat{\vartheta}) \Sigma_{n}(\hat{\vartheta}) H_{n}^{-1}(\hat{\vartheta}), \text { where } \Sigma_{n}(\vartheta)=\sum_{t=1}^{n} \frac{\partial \ell_{t}(\vartheta)}{\partial \vartheta} \frac{\partial \ell_{t}(\vartheta)}{\partial \vartheta^{\prime}}
$$

and $H_{n}(\vartheta)$ is defined in eq. (10). As we shall see, $\hat{\Omega}$ automatically adjusts to the level of persistence and converges to the correct asymptotic limit in both cases. As a consequence, for example, standard $t$-statistic will be normally distributed in large samples whether $x_{t}$ is stationary or nonstationary:

Theorem 11 Under either Assumptions 1-2 or Assumption 3, with $\hat{\Omega}$ defined in eq. (19),

$$
t:=\hat{\Omega}^{-1 / 2}\left\{\hat{\vartheta}-\vartheta_{0}\right\} \rightarrow{ }_{d} N\left(0, I_{4}\right)
$$

This result shows that standard inferential procedures regarding $\vartheta_{0}$ are robust to the persistence of $x_{t}$. We conjecture that similar results hold for other statistics such as the likelihood-ratio statistic.

\section{Simulation Study}

To investigate the relevance and usefulness of our asymptotic results, we conduct a simulation study to see whether standard $t$-statistics are sensitive towards the level of persistence, $d_{x}$, in finite samples. Our simulation design is based on the GARCH-X model with the exogenous regressor $x_{t}$ being generated by $x_{t}=(1-L)^{-d_{x}} v_{t}$. The data-generating GARCH parameter values are set to be $\omega_{0}=0.01, \alpha_{0}=0.05, \beta_{0}=0.6$ and $\pi_{0}=0.1$. These parameter values are similar to the estimates reported in Shephard and Sheppard (2010) where $x_{t}^{2}$ is a realized volatility measure. The innovation processes $\left\{\varepsilon_{t}\right\}$ and $\left\{v_{t}\right\}$ are chosen to be i.i.d. standard normal and mutually independent. ${ }^{3}$ The initial values are set $x_{0}=0$ and $\sigma_{0}^{2}=0.01$. We consider the following four data generating processes depending on $d_{x}$ in $x_{t}$ :

\begin{tabular}{llll}
\hline \multicolumn{2}{l}{ stationary cases } & \multicolumn{2}{c}{ nonstationary cases } \\
\hline DGP 1 & $d_{x}=0.0$ & DGP 3 & $d_{x}=0.7$ \\
DGP 2 & $d_{x}=0.3$ & DGP 4 & $d_{x}=1.0$ \\
\hline
\end{tabular}

The null distributions of each of the $t$-statistics associated with $\omega, \alpha, \beta$ and $\pi$ are simulated for $n=500$ and 5,000 with 10,000 iterations. The simulation results are reported in Figures 1 and 2. Figure 1 reports the results for the stationary cases and show that the large sample $N(0,1)$ distribution of the $t$-statistics is a very good finite-sample approximation. For the non-stationary cases as reported in Figure 2, the asymptotic $N(0,1)$ approximation is also precise, albeit less so compared to the stationary case.

\footnotetext{
${ }^{3}$ We also tried the case for $v_{t}=-\varepsilon_{t}$ and the results are still similar.
} 
Figure 1. The simulated distributions of $t$-statistics for the stationary cases DGP 1: $d x=0.0, n=500$

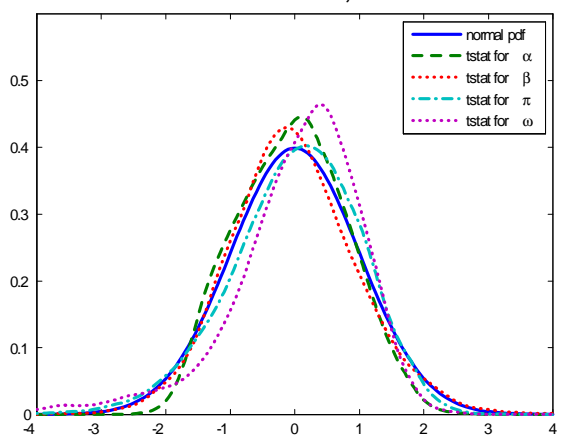

DGP 1: $\mathrm{dx}=0.0, \mathrm{n}=5000$
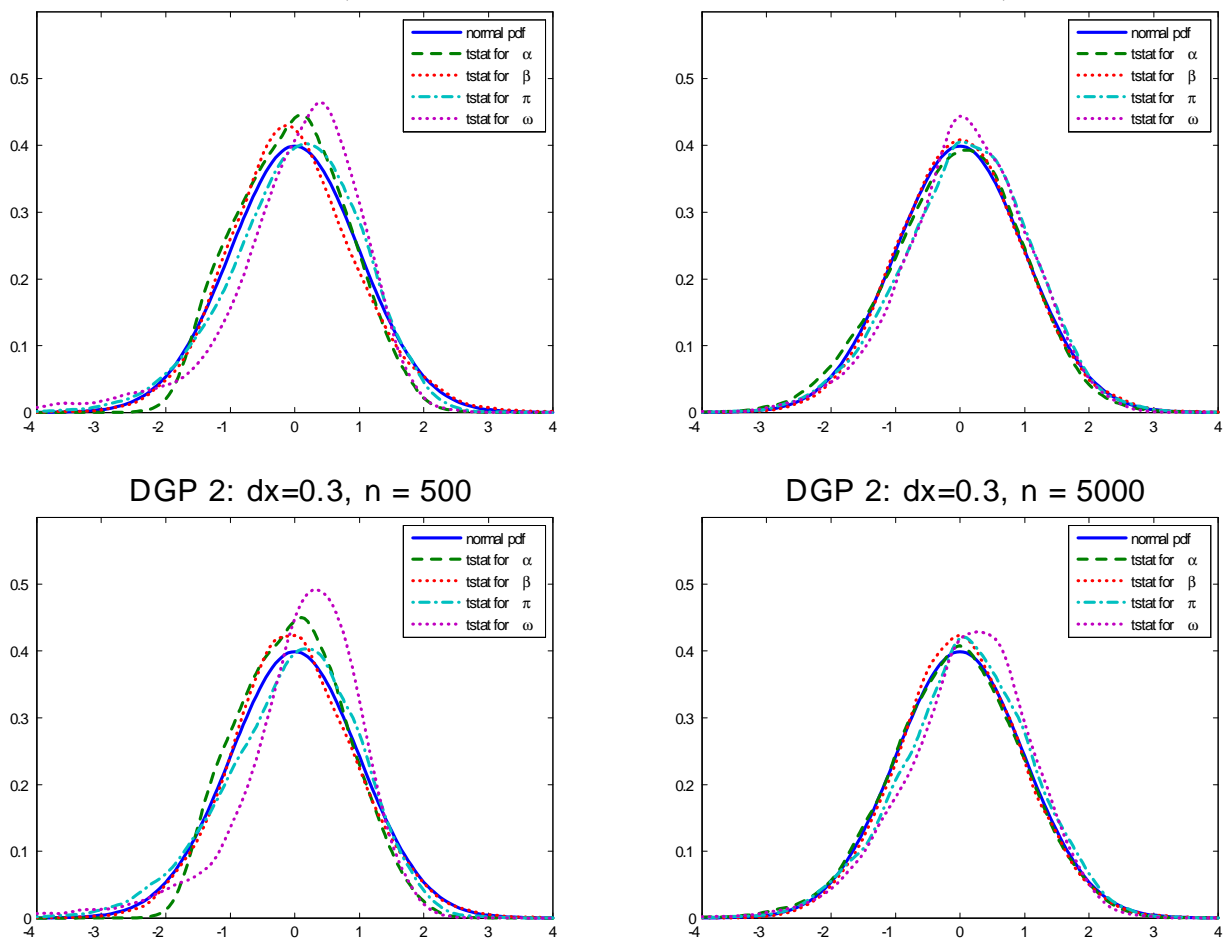

Figure 2. The simulated distributions of $t$-statistics for the nonstationary cases DGP 3: $d x=0.7, n=500$

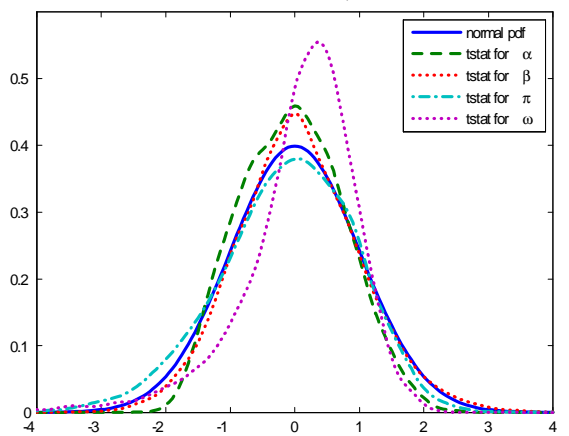
DGP 3: $d x=0.7, n=5000$
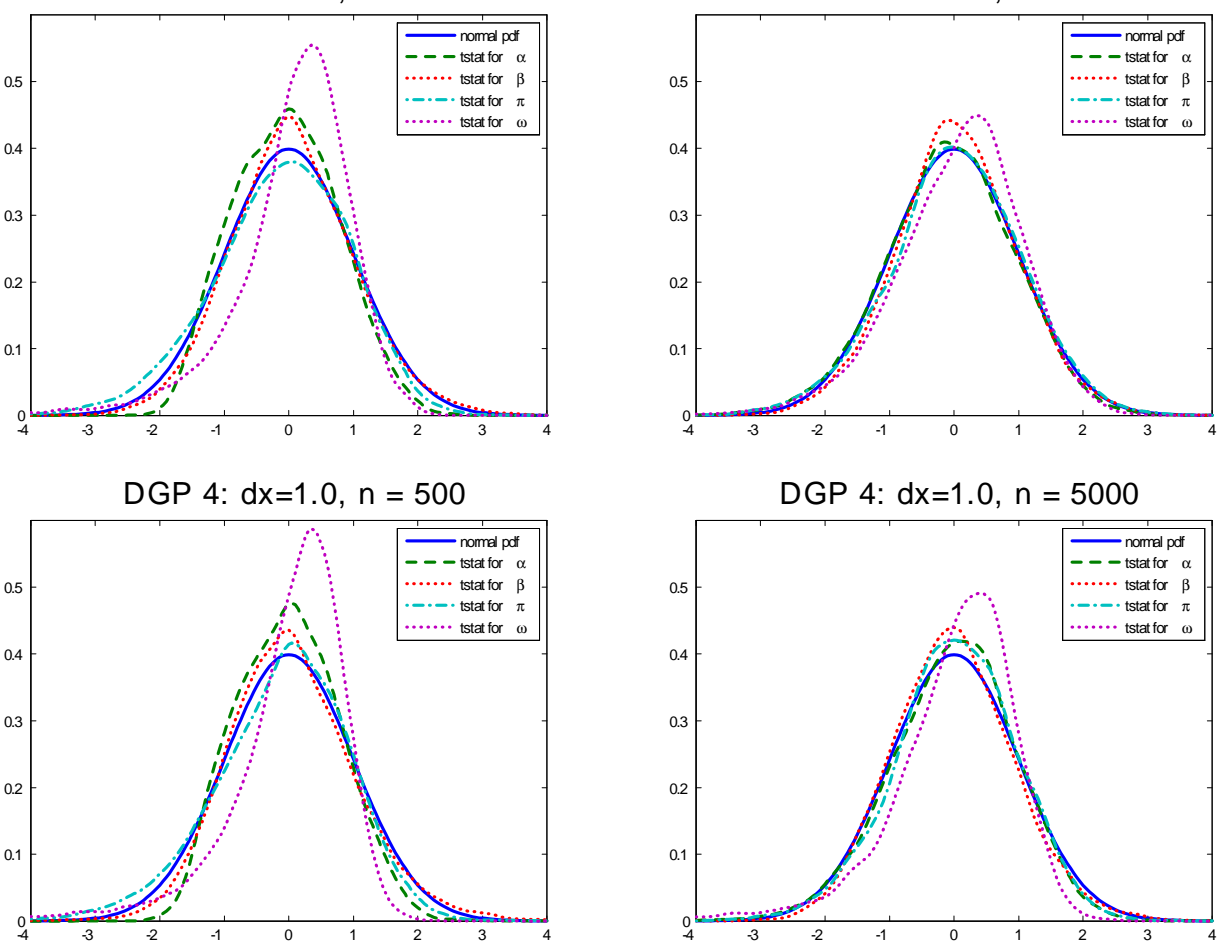
The results for the $t$-statistic associated with $\omega$ are consistent with theory: We found that $\hat{\omega}$ will converge towards its limiting distribution at a slower rate compared to $\hat{\theta}$ when the regressor is persistent. This is reflected in the finite-sample distributions of its $t$-statistic reported in Figures 1-2: As persistence grows, the precision of the asymptotic approximation for the distribution of $\omega$ 's $t$-statistic deterioriates compared to the other $t$-statistics for any given sample size.

Our simulation results show that the empirical distributions of the $t$-statistics are close to normal for moderate sample sizes and become more so as the sample size increases. This is true regardless of the value of the memory parameter $d_{x}$ in $x_{t}$. In conclusion, the individual t-statistics of $(\omega, \alpha, \beta, \pi)$ are robust towards the dependence structure of $x_{t}$ in the GARCH-X model. Researchers do not need to determine whether $x_{t}$ is stationary or not before they implement the QMLE and associated inferential tools for the GARCH-X model.

\section{Conclusion}

We have here developed asymptotic theory of QMLE's in GARCH models with additional persistent covariates in the variance specification. It is shown that the asymptotic behaviour of the QMLE's depend on whether the regressor is stationary or not. At the same time, standard inferential tools, such as $t$-statistics, for the parameters are robust towards the level of persistence. In particular, in contrast to the explosive case in pure GARCH models, one can draw inference about the intercept parameter $\omega$.

A number of extensions of the theory would be of interest: For example, to show global consistency of $\hat{\omega}$ and to analyze the properties of the QMLE in alternative GARCH specifications with persistent regressors.

\section{Acknowledgments}

This paper benefited from discussions with Joon Y. Park, Peter Phillips, Anders Rahbek and Qiying Wang. We also wish to thank participants at seminars at University of Nottingham, the NBER-NSF time series conference 2012 at Texas A\&M, and a time series workshop at University of Copenhagen for comments and suggestions. Kristensen gratefully acknowledges financial support from the European Research Council through a starting grant (No 312474), Economic and Social Research Council through the ESRC Centre for Microdata Methods and Practice grant RES-589-280001, and the Danish Research Council through its funding of CREATES. This work was supported by a grant from the Kyung Hee University (KHU 2012-1676).

\section{A Proofs of Theorems}

Proof of Theorem 3. Define $\hat{\vartheta}^{*}=\arg \max _{\vartheta \in \Theta \times \mathcal{W}} L_{n}^{*}(\vartheta)$ where $L_{n}^{*}(\vartheta)$ is defined in eq. (9) with $r_{t}(\vartheta)$ given in eq. (16). We first show consistency of $\hat{\vartheta}^{*}$ by verifying the conditions in Kristensen and Rahbek (2005, Proposition 2): (i) The parameter space $\Theta \times \mathcal{W}$ is a compact 
Euclidean space with $\vartheta_{0} \in \Theta \times \mathcal{W}$; (ii) $\vartheta \mapsto \ell_{t}^{*}(\vartheta)$ is continuous almost surely; (iii) $L_{n}^{*}(\vartheta) / n \rightarrow p$ $L^{*}(\vartheta):=\mathbb{E}\left[\ell_{t}^{*}(\vartheta)\right]$ where the limit exists, $\forall \vartheta \in \Theta \times \mathcal{W}$; (iv) $L^{*}\left(\vartheta_{0}\right)>L^{*}(\vartheta), \forall \vartheta \neq \vartheta_{0}$; and (v) $\mathbb{E}\left[\sup _{\vartheta \in \Theta \times \mathcal{W}} \ell_{t}^{*}(\vartheta)\right]<+\infty$. Condition (i) holds by assumption, while (ii) follows by the continuity of $\vartheta \mapsto r_{t}^{2}(\vartheta)$ as given in eq. (16). Condition (iii) follows by the LLN for stationary and ergodic sequences if the limit $L^{*}(\vartheta)$ exists; the limit is indeed well-defined since $\ell_{t}^{*}(\vartheta) \leq-\log \left(\omega / \omega_{0}\right)$ such that $\mathbb{E}\left[\ell_{t}^{*}(\vartheta)^{+}\right]<\infty$. To prove condition (iv), first observe that $r_{t}^{*}\left(\vartheta_{0}\right)=1$ which in turn implies that $L^{*}\left(\vartheta_{0}\right)=0$. Moreover, $\omega_{0} \leq \log \left(\sigma_{0, t}^{2}\left(\vartheta_{0}\right)\right)$ such that $\mathbb{E}\left[\left(\log \sigma_{0, t}^{2}\left(\vartheta_{0}\right)\right)^{-}\right]<\infty$, while $\mathbb{E}\left[\left(\log \sigma_{0, t}^{2}\left(\vartheta_{0}\right)\right)^{+}\right] \leq\left(\log \mathbb{E}\left[\sigma_{0, t}^{2 s}\left(\vartheta_{0}\right)\right]\right)^{+} / s<\infty$ by Jensen's inequality and Lemma 2 . Thus, $\mathbb{E}\left[\left|\ell_{t}^{*}\left(\vartheta_{0}\right)\right|\right]<\infty$ is well-defined, while either (a) $L^{*}(\vartheta)=-\infty$ or $(\mathrm{b}) L^{*}(\vartheta) \in(-\infty, \infty)$. Now, let $\vartheta \neq \vartheta_{0}$ be given: Then, if (a) holds, $L^{*}\left(\vartheta_{0}\right)>-\infty=L^{*}(\vartheta)$. If (b) holds, the following calculations are allowed:

$$
L^{*}(\vartheta)=-\mathbb{E}\left[\log \left(r_{t}^{*}(\vartheta)\right)+\left\{\frac{1}{r_{t}^{*}(\vartheta)}-1\right\} \varepsilon_{t}^{2}\right]=-\mathbb{E}\left[\log \left(r_{t}^{*}(\vartheta)\right)+\left\{\frac{1}{r_{t}^{*}(\vartheta)}-1\right\}\right],
$$

where we have used that $\mathbb{E}\left[\varepsilon_{t}^{2} \mid \mathcal{F}_{t-1}\right]=1$. Thus, $L^{*}(\vartheta) \leq 0=L^{*}\left(\vartheta_{0}\right)$ with equality if and only if $r_{t}^{2}(\vartheta)=1$ a.s. Suppose that $r_{t}^{2}(\vartheta)=1$ a.s. $\Leftrightarrow \sigma_{0, t}^{2}(\vartheta)=\sigma_{0, t}^{2}\left(\vartheta_{0}\right)$ a.s. With $c_{i}(\theta):=$ $\left(\alpha \beta^{i-1}, \pi \beta^{i-1}\right)^{\prime}$, we then claim that $\omega_{0}=\omega$ and $c_{i}\left(\theta_{0}\right)=c_{i}(\theta)$ for all $i \geq 1$; this in turn implies $\vartheta=\vartheta_{0}$. We show this by contradiction: Let $m>0$ be the smallest integer for which $c_{i}\left(\theta_{0}\right) \neq c_{i}(\theta)$ (if $c_{i}\left(\theta_{0}\right)=c_{i}(\theta)$ for all $i \geq 1$, then $\omega_{0}=\omega$ ). Thus,

$$
a_{0} y_{t-m}^{2}+b_{0} x_{t-m}^{2}=\omega-\omega_{0}+\sum_{i=1}^{\infty} a_{i} y_{t-m-i}^{2}+\sum_{i=1}^{\infty} b_{i} x_{t-m-i}^{2}
$$

where $a_{i}:=\alpha_{0} \beta_{0}^{i-1}-\alpha \beta^{i-1}$ and $b_{i}:=\pi_{0} \beta_{0}^{i-1}-\pi \beta^{i-1}$. The right hand side belongs to $\mathcal{F}_{t-m-1}$ and so $a_{0} y_{t-m}^{2}+b_{0} x_{t-m}^{2} \mid \mathcal{F}_{t-m-1}$ is constant. This is ruled out by Assumption 1(iv). Finally, condition (v) follows from $\sup _{\vartheta \in \Theta \times \mathcal{W}} \ell_{t}^{*}(\vartheta) \leq-\sup _{\vartheta \in \Theta \times \mathcal{W}} \log (\omega) \leq-\log (\underline{\omega})<+\infty$.

Now, return to the actual, feasible QMLE, $\hat{\vartheta}$. Using Lemma 2 ,

$$
\sup _{\vartheta \in \mathcal{W} \times \Theta}\left|L_{n}^{*}(\vartheta)-L_{n}(\vartheta)\right| \leq \frac{K}{\underline{\omega}^{2}} \sum_{t=1}^{n} \bar{\beta}^{t} y_{t-1}^{2}+\frac{K}{\underline{\omega}^{2}} \sum_{t=1}^{n} \bar{\beta}^{t}
$$

where $\lim _{n \rightarrow \infty} \sum_{t=1}^{n} \bar{\beta}^{t}=(1-\bar{\beta})^{-1}<\infty$ while $\lim _{n \rightarrow \infty} \sum_{t=1}^{n} \bar{\beta}^{t} y_{t-1}^{2}<\infty$ by Berkes et al (2003, Lemma 2.2) in conjunction with Lemma 1. Thus, $\sup _{\vartheta \in \Theta}\left|L_{n}^{*}(\vartheta)-L_{n}(\vartheta)\right| / n=o_{p}(1 / n)$. Combining this with the above analysis of $L_{n}^{*}(\vartheta)$, it then follows from Kristensen and Shin (2012, Proposition 1) that $\left\|\hat{\vartheta}^{*}-\hat{\vartheta}\right\|=o_{p}(1 / n)$. In particular, $\hat{\vartheta}$ is consistent.

Proof of Theorem 5. As shown in the proof of Theorem $3,\left\|\hat{\vartheta}^{*}-\hat{\vartheta}\right\|=o_{p}(1 / \sqrt{n})$; thus, it suffices to analyze $\hat{\vartheta}^{*}$. The score and hessian are given by

$$
S_{n}^{*}(\vartheta)=\frac{\partial L_{n}^{*}(\vartheta)}{\partial \vartheta}=\sum_{t=1}^{n} \frac{1}{\sigma_{0, t}^{2}(\vartheta)} \frac{\partial \sigma_{0, t}^{2}(\vartheta)}{\partial \vartheta}\left\{\frac{y_{t}^{2}}{\sigma_{0, t}^{2}(\vartheta)}-1\right\}, \quad H_{n}^{*}(\vartheta)=\frac{\partial^{2} L_{n}^{*}(\vartheta)}{\partial \vartheta \partial \vartheta^{\prime}}=\sum_{t=1}^{n} h_{t}^{*}(\vartheta),
$$


where derivatives w.r.t. $\sigma_{0, t}^{2}(\vartheta)$ can be found in the proof of Lemma 4 , and

$$
\begin{aligned}
h_{t}^{*}(\vartheta)= & \left\{\frac{1}{\sigma_{0, t}^{2}(\vartheta)} \frac{\partial^{2} \sigma_{0, t}^{2}(\vartheta)}{\partial \vartheta \partial \vartheta^{\prime}}-\frac{1}{\sigma_{0, t}^{4}(\vartheta)} \frac{\partial \sigma_{0, t}^{2}(\vartheta)}{\partial \vartheta} \frac{\partial \sigma_{0, t}^{2}(\vartheta)}{\partial \vartheta^{\prime}}\right\}\left\{\frac{y_{t}^{2}}{\sigma_{0, t}^{2}(\vartheta)}-1\right\} \\
& -\frac{\partial \sigma_{0, t}^{2}(\vartheta)}{\partial \vartheta} \frac{\partial \sigma_{0, t}^{2}(\vartheta)}{\partial \vartheta^{\prime}} \frac{y_{t}^{2}}{\sigma_{0, t}^{6}(\vartheta)} .
\end{aligned}
$$

We now verify the the two convergence results stated in eq. (11): First, we employ the CLT for Martingale differences in Brown (1971, Theorem 2) to show that the first part of eq. (11) holds. By Assumption 1(i), $X_{t}:=\partial r_{t}^{*}\left(\vartheta_{0}\right) /(\partial \vartheta)\left\{\varepsilon_{t}^{2}-1\right\}$ is a Martingale difference and $S_{n}^{*}\left(\vartheta_{0}\right) / \sqrt{n}$ has quadratic variation

$$
\left\langle S_{n}^{*}\left(\vartheta_{0}\right) / \sqrt{n}\right\rangle=\kappa_{4} \frac{1}{n} \sum_{t=1}^{n} \frac{\partial r_{t}^{*}\left(\vartheta_{0}\right)}{\partial \vartheta} \frac{\partial r_{t}^{*}\left(\vartheta_{0}\right)}{\partial \vartheta^{\prime}} \rightarrow_{p} \kappa_{4} \mathbb{E}\left[\frac{\partial r_{t}^{*}\left(\vartheta_{0}\right)}{\partial \vartheta} \frac{\partial r_{t}^{*}\left(\vartheta_{0}\right)}{\partial \vartheta^{\prime}}\right]<\infty
$$

where we have used Assumption 2(i) and Lemma 4. This shows that eq. (1) in Brown (1971) holds. By stationarity and $\mathbb{E}\left[\left\|X_{t}\right\|^{2}\right]<\infty, \sum_{t=1}^{n} \mathbb{E}\left[\left\|X_{t}\right\|^{2} \mathbb{I}\left\{\left\|X_{t}\right\|>c \sqrt{n}\right\}\right] / n=\mathbb{E}\left[\left\|X_{t}\right\|^{2} \mathbb{I}\left\{\left\|X_{t}\right\|>c \sqrt{n}\right\}\right] \rightarrow$ 0 , and so eq. (2) of Brown (1971) also holds.

For the hessian, $\left\|h_{t}^{*}(\vartheta)\right\| \leq\left\{B_{2, t}+B_{1, t}^{2}\right\}\left\{1+B_{0, t} \varepsilon_{t}^{2}\right\}+B_{1, t}^{2} B_{0, t} \varepsilon_{t}^{2}$ for all $\vartheta$ in some neighbourhood of $\vartheta_{0}$, where the right-hand side has finite first moment, c.f. Lemma 4 . It now follows by standard uniform convergence results for averages of stationary sequences (see e.g. Kristensen and Rahbek (2005, Proposition 1) that $\sup _{\left\|\vartheta-\vartheta_{0}\right\|<\delta}\left\|H_{n}^{*}(\vartheta)-H^{\text {st }}(\vartheta)\right\| \rightarrow_{p} 0$, for some $\delta>0$, where $H^{\text {stat }}(\vartheta)=\mathbb{E}\left[h_{\vartheta \vartheta, t}^{*}(\vartheta)\right]$. Moreover, $\vartheta \mapsto H^{\text {st }}(\vartheta)$ is continuous. Since $\hat{\vartheta}^{*} \rightarrow_{p} \vartheta_{0}, \bar{\vartheta} \rightarrow_{p} \vartheta_{0}$ and so lies in any arbitrarily small neighbourhood w.p.a.1. To complete the proof, we verify that $H_{\vartheta \vartheta}^{\text {st }}\left(\vartheta_{0}\right)$ is non-singular: The process $\Psi_{t}:=\partial \sigma_{0, t}^{2}\left(\vartheta_{0}\right) /(\partial \vartheta) \in \mathbb{R}^{4}$ can be written as $\Psi_{t}=\beta \Psi_{t-1}+W_{t}$, where $W_{t}:=\left[1, y_{t-1}, x_{t-1}, \sigma_{0, t-1}^{2}\left(\vartheta_{0}\right)\right]^{\prime}$. Suppose that there exists $\lambda \in \mathbb{R}^{4} \backslash\{0\}$ and $t \geq 1$ such that $\lambda^{\prime} \Psi_{t}=0$ a.s. Since $\Psi_{t}$ is stationary, this must hold for all $t$. This implies that $\lambda^{\prime} W_{t}=0$ a.s. for all $t \geq 1$. However, this is ruled out by Assumption 1(iv). It must therefore hold that $\lambda^{\prime} \Psi_{t} / \sigma_{0, t}^{2}\left(\vartheta_{0}\right)=0$ if and only if $\lambda=0$; thus, $H^{\text {st }}\left(\vartheta_{0}\right)=\mathbb{E}\left[\Psi_{t} \Psi_{t}^{\prime} / \sigma_{0, t}^{4}\left(\vartheta_{0}\right)\right]$ is non-singular.

Proof of Theorem 6. To prove (i), define $\psi_{n}^{\prime}(s)=n^{-(1 / 2-d)} \sum_{t=1}^{[n s]} f\left(x_{t-1}\right) w_{t}$ and $\psi_{n}^{\prime \prime}(s)=$ $n^{-(1 / 2-d)} \sum_{t=1}^{[n s]} f\left(x_{t-1}\right) \mathbb{E}\left[w_{t}\right]$ which both belong to $D[0,1]$. First, by Theorem 2.1 in Wang and Phillips (2009a), henceforth WP2009a, and Lemma 1 in Kasparis et. al. (2012), $\psi_{n}^{\prime \prime}(s) \Rightarrow$ $L_{W_{d}}(s, 0) \times K \mathbb{E}\left[w_{t}\right] \int_{-\infty}^{\infty} f(x) d x$ on $D[0,1]$. We show the following two claims: (i.a) $\left|\psi_{n}^{\prime}(s)-\psi_{n}^{\prime \prime}(s)\right|=$ $o_{p}(1)$ and (i.b) $\psi_{n}^{\prime}(s)$ is tight; (i.a) implies that $\psi_{n}^{\prime}(s)$ and $\psi_{n}^{\prime \prime}(s)$ have the same finite dimensional limit distributions which together with (i.b) imply weak convergence of $\psi_{n}^{\prime}(s)$ towards the limit of $\psi_{n}^{\prime \prime}(s)$. To show (i.a), use independence between $w_{t}$ and $x_{t}$ to write with $\mathcal{X}_{n}=\left(x_{1}, \ldots, x_{n}\right)$,

$$
\mathbb{E}\left[\left|\psi_{n}^{\prime}(s)-\psi_{n}^{\prime \prime}(s)\right|^{2} \mid \mathcal{X}_{n}\right]=\frac{\operatorname{Var}\left(w_{t}\right)}{n^{2(1 / 2-d)}} \sum_{t=1}^{n} f^{2}\left(x_{t-1}\right)+\frac{1}{n^{2(1 / 2-d)}} \sum_{t \neq u} f\left(x_{t-1}\right) f\left(x_{u-1}\right) \operatorname{Cov}\left(w_{t}, w_{u}\right) .
$$


Using the covariance condition together with $|f(x)| \leq C$ for some $C<\infty$, we obtain

$$
\left|\sum_{t \neq u} f\left(x_{t-1}\right) f\left(x_{u-1}\right) \operatorname{Cov}\left(w_{t}, w_{u}\right)\right| \leq C \sum_{t=1}^{n}\left|f\left(x_{t-1}\right)\right| \times \sum_{u=1}^{\infty}\left|\operatorname{Cov}\left(w_{0}, w_{u}\right)\right| .
$$

By WP2009a, $n^{-1 / 2+d} \sum_{t=1}^{n}\left|f\left(x_{t-1}\right)\right|^{q}=O_{p}(1), q=1,2$, and so $\mathbb{E}\left[\left|\psi_{n}^{\prime}(s)-\psi_{n}^{\prime \prime}(s)\right|^{2} \mid \mathcal{X}_{n}\right]=o_{p}(1)$. By Markov's inequality, this implies that $P\left(\left|\psi_{n}^{\prime}(s)-\psi_{n}^{\prime \prime}(s)\right|^{2}>\delta \mid \mathcal{X}_{n}\right)=o_{p}(1)$ for any $\delta>0$. Thus, $P\left(\left|\psi_{n}^{\prime}(s)-\psi_{n}^{\prime \prime}(s)\right|^{2}>\delta\right)=\mathbb{E}\left[P\left(\left|\psi_{n}^{\prime}(s)-\psi_{n}^{\prime \prime}(s)\right|^{2}>\delta \mid \mathcal{X}_{n}\right)\right] \rightarrow 0$. To show (i.b), we apply Theorem 5 in Billingsley (1974) and wish to show that there exists a sequence of $\alpha_{n}(\epsilon, \delta)$ satisfying $\lim _{\delta \rightarrow 0} \lim \sup _{n \rightarrow \infty} \alpha_{n}(\epsilon, \delta)=0$ for each $\epsilon>0$ such that, for $0 \leq s_{1} \leq \cdots \leq s_{m} \leq s \leq 1, s-s_{m} \leq \delta$, we have

$$
P\left(\left|\psi_{n}^{\prime}(s)-\psi_{n}^{\prime}\left(s_{m}\right)\right| \geq \epsilon \mid \psi_{n}^{\prime}\left(s_{1}\right), \psi_{n}^{\prime}\left(s_{2}\right), \cdots, \psi_{n}^{\prime}\left(s_{m}\right)\right) \leq \alpha_{n}(\epsilon, \delta), \quad \text { a.s. }
$$

A sufficient conditions for eq. (20) is

$$
\sup _{\left|s_{1}-s_{2}\right| \leq \delta} P\left(\left|\sum_{t=\left[n s_{1}\right]+1}^{\left[n s_{2}\right]} f\left(x_{t-1}\right) w_{t}\right| \geq \epsilon n^{1 / 2-d} \mid \psi_{n}^{\prime}\left(s_{1}\right), \psi_{n}^{\prime}\left(s_{2}\right), \cdots, \psi_{n}^{\prime}\left(s_{m}\right)\right) \leq \alpha_{n}(\epsilon, \delta) .
$$

As before, we first establish a conditional version: Define $\alpha_{n}\left(\mathcal{X}_{n}, \epsilon, \delta\right)$ as

$$
\alpha_{n}\left(\mathcal{X}_{n}, \epsilon, \delta\right):=\epsilon^{-2} n^{-2(1 / 2-d)} \sup _{0 \leq s \leq \delta} \mathbb{E}\left[\left\{\sum_{t=1}^{[n s]} f\left(x_{t-1}\right) w_{t}\right\}^{2} \mid \mathcal{X}_{n}\right]
$$

Similar to the proof of (i.a), we have that, for large enough $n$,

$$
\begin{aligned}
\alpha_{n}\left(\mathcal{X}_{n}, \epsilon, \delta\right) & \leq \epsilon^{-2} n^{-2(1 / 2-d)} \sum_{t=1}^{n} f^{2}\left(x_{t-1}\right) \mathbb{E}\left[w_{t}^{2}\right]+\epsilon^{-2} n^{-2(1 / 2-d)} \sum_{t_{1} \neq t_{2}} f\left(x_{t_{1}-1}\right) f\left(x_{t_{2}-1}\right)\left|\mathbb{E}\left[w_{t_{1}} w_{t_{2}}\right]\right| \\
& \leq \epsilon^{-2} n^{-(1 / 2-d)} O_{p}(1)
\end{aligned}
$$

This shows that eq. (20) holds in probability conditional on $\mathcal{X}_{n}$ which in turn implies that it also holds unconditionally of $\mathcal{X}_{n}$.

To show (ii), write $n^{d / 2-1 / 4} \sum_{t=1}^{[n s]} f\left(x_{t-1}\right) w_{t} u_{t}=\sum_{t=1}^{[n s]} Z_{n, t} w_{t} u_{t}$ where $Z_{n, t}:=n^{-(1 / 4-d / 2)} f\left(x_{t-1}\right)$. The sequence $\left\{Z_{n, t} w_{t} u_{t}\right\}$ is a martingale difference w.r.t. $\mathcal{F}_{t}$ with quadratic variation, $\sigma_{u}^{2} \sum_{t=1}^{[n s]} Z_{n, t}^{2} w_{t}^{2}$. By the same arguments as in the proof of part (i) of this lemma, $\sigma_{u}^{2} \sum_{t=1}^{[n s]} Z_{n, t}^{2} w_{t}^{2}=\Lambda_{n}(s)+o_{p}(1)$ where $\Lambda_{n}(s)=\sigma_{u}^{2} \mathbb{E}\left[w_{t}^{2}\right] \times \sum_{t=1}^{[n s]} Z_{n, t}^{2} \Rightarrow K \sigma_{u}^{2} \mathbb{E}\left[w_{t}^{2}\right] \int_{-\infty}^{\infty} f^{2}(x) d x \times L_{W_{d}}(s, 0)$. As in Proof of Theorem 3.1 in WP2009a, under a suitable probability space there exists an equivalent process $x_{t}^{*}$ of $x_{t}$ such that the corresponding quadratic variation $\Lambda_{n}^{*}(s) \rightarrow{ }_{p} K \sigma_{u}^{2} \mathbb{E}\left[w_{t}^{2}\right] \int_{-\infty}^{\infty} f^{2}(x) d x \times L_{W_{d}}(s, 0)$. Without loss of generality we assume that $x_{t}$ satisfies this. We now wish to show that $V_{n}(s):=$ $\Lambda_{n}^{-1 / 2}(s) \sum_{t=1}^{[n s]} Z_{n, t} w_{t} u_{t} \Rightarrow G(s)$ on $D[0,1]$, where $G(s)$ is a Gaussian process with covariance kernel $\left(s_{1} \wedge s_{2}\right)$ along the lines of the proof of eq. 5.21 in WP2009a: First, observe that since $\left\{x_{t}\right\}$, and 
therefore $\Lambda_{n}^{2}(s)$, is independent of $\left\{w_{t}, u_{t}\right\}, V_{n}(s)$ is a martingale conditional on $\mathcal{X}_{n}$. It then follows from Hall and Heyde (1981, Theorem 3.9) that $\sup _{v}\left|P\left(V_{n}(s) \leq v \mid \mathcal{X}_{n}\right)-\Phi(v)\right| \leq A\left(q_{u}\right) \mathcal{L}_{n}^{1 /\left(1+q_{u}\right)}$ a.s., for any $s \in[0,1]$, where $A\left(q_{u}\right)$ is a constant depending only on $q_{u}$ and

$$
\mathcal{L}_{n}=\frac{\sup _{t \geq 1} \mathbb{E}\left[\left|u_{t} w_{t}\right|^{q_{u}}\right]}{\Lambda_{n}^{q_{u}}} \sum_{t=1}^{n}\left|Z_{n, t}\right|^{q_{u}}+\frac{\sigma_{u}^{q_{u}}}{\Lambda_{n}^{q_{u}}} \mathbb{E}\left[\left|\sum_{t=1}^{n} Z_{n, t}^{2}\left\{w_{t}^{2}-\mathbb{E}\left[w_{t}^{2}\right]\right\}\right|^{q_{u} / 2} \mid \mathcal{X}_{n}\right]
$$

By part (i), $\sum_{t=1}^{n}\left|Z_{n, t}\right|^{q_{u}}=o_{p}(1)$ and so the first term is $o_{p}$ (1). As before, assuming without loss of generality $q_{u} \leq 4, \mathbb{E}\left[\left|\sum_{k=1}^{n} f^{2}\left(x_{t-1}\right)\left\{w_{t}^{2}-\mathbb{E}\left[w_{t}^{2}\right]\right\}\right|^{2} \mid \mathcal{X}_{n}\right] \leq C \sum_{t=1}^{n} f^{2}\left(x_{t-1}\right) \times \sum_{u=1}^{\infty}\left|\operatorname{Cov}\left(w_{t}^{2}, w_{u}^{2}\right)\right|$, and so the second term of $\mathcal{L}_{n}$ is also $o_{p}(1)$. We conclude that $\sup _{v}\left|P\left(V_{n}(s) \leq v\right)-\Phi(v)\right| \leq$ $\mathbb{E}\left[\sup _{v}\left|P\left(V_{n}(s) \leq v \mid \mathcal{X}_{n}\right)-\Phi(v)\right|\right] \rightarrow 0$. Finally, tightness of $V_{n}(s)$ follows by the same arguments as in the proof of (i).

Proof of Theorem 9. We first show that $\hat{\theta}^{*}:=\arg \max _{\theta \in \Theta} L_{n}^{*}(\theta)$ satisfies $\hat{\theta}^{*} \rightarrow^{P} \theta_{0}$. This is shown by verifying conditions (i)-(v) as stated in the proof of Theorem 3. Condition (i) holds by assumption, while (ii) follows by the continuity of $\theta \mapsto r_{t}^{*}(\theta)$ as given in eq. (18). Condition (iii) follows by the LLN for stationary and ergodic sequences if the limit $L^{*}(\vartheta)$ exists; the limit is indeed well-defined since, by Lemma $8, \mathbb{E}\left[r_{t}^{*}(\theta)^{-k}\right]<\infty$ for any $k>0$. To prove condition (iv), we see that, by the same arguments as in the proof of Theorem $5, L^{*}\left(\theta_{0}\right) \geq L^{*}(\theta)$ with equality if and only if $r_{t}^{*}(\theta)=1$ a.s. Suppose that indeed $r_{t}^{*}(\theta)=1$ a.s. for some $\theta \in \Theta$. By definition of $r_{t}(\theta)$, this is equivalent to $z_{t}(\theta)=z_{t}$ a.s.,where $z_{t}(\theta)$ is defined in eq. (18). Observe that with $\tilde{y}_{t}=z_{t} \varepsilon_{t}$, we have that the two processes satisfy $z_{t}=1+\alpha_{0} \tilde{y}_{t-1}^{2}+\beta_{0} z_{t-1}$ and $z_{t}(\theta)=\pi / \pi_{0}+\alpha \tilde{y}_{t-1}^{2}+\beta z_{t-1}(\theta)$. Thus, the processes correspond to the true and model-implied volatility in a pure GARCH model with intercept $\tilde{\omega}=\pi / \pi_{0}$. We can then employ the same arguments as in the proof of Theorem 3 to show that $z_{t}(\theta)=z_{t}$ a.s. $\Leftrightarrow \theta=\theta_{0}$. Finally, condition (v) follows from

$$
\left|\ell_{t}^{*}(\theta)\right| \leq\left|\log r_{t}^{*}(\theta)\right|+\varepsilon_{t}^{2}\left\{\frac{1}{r_{t}(\theta)}+1\right\} \leq \sup _{\theta \in \Theta} r_{t}^{*}(\theta)^{s}+\sup _{\theta \in \Theta} r_{t}^{*}(\theta)^{-1}+\varepsilon_{t}^{2}\left\{\sup _{\theta \in \Theta} r_{t}^{*}(\theta)^{-1}+1\right\}=: \bar{\ell}_{t}^{*},
$$

where $\mathbb{E}\left[\bar{\ell}_{t}^{*}\right]<\infty$ by Lemma 8 .

Now, return to the original estimator, $\hat{\vartheta}$. Write the log-likelihood as $L_{n}(\vartheta)=L_{n}^{*}(\theta)+R_{n}(\vartheta)$, where $R_{n}(\vartheta)=\sum_{t=1}^{n}\left[\varepsilon_{t}^{2}\left\{1 / r_{t}^{*}(\theta)-11 / r_{t}(\vartheta)\right\}+\log \left(r_{t}^{*}(\theta) / r_{t}(\vartheta)\right)\right] / n$. Using the same arguments as in Francq and Zakoian $\left(2012\right.$, p. 844) together with Lemma 8, we obtain that $R_{n}(\vartheta)=o_{p}(1)$ uniformly in $\vartheta$. Thus, by the same arguments as in the proof of Theorem $3,\left\|\hat{\vartheta}-\hat{\vartheta}^{*}\right\|=o_{p}(1)$ where $\hat{\vartheta}^{*}=\arg \max _{\theta \in \Theta} \tilde{L}_{n}^{*}(\vartheta)$ and $\tilde{L}_{n}^{*}(\omega, \theta)=L_{n}^{*}(\theta)$ for any $(\omega, \theta) \in \mathcal{W} \times \Theta$.

Local consistency of $\hat{\omega}$ and the local rate result for $\hat{\theta}$ follow as part of the results shown in the proof of Theorem 10 together with Kristensen and Rahbek (2010, Lemma 11).

Proof of Theorem 10. We first establish some approximations: It follows from Lemma 8 that $\beta^{i-1} w_{n \xi}^{-2} \sigma_{t-i}^{2}=\beta^{i-1}\left(w_{n \xi}^{-2} \pi_{0} x_{t-1}^{2}\right) z_{t-i}+o_{p}(1)$, for all $i \geq 1$ and $t=1, \ldots, n$, and note that $\max _{1 \leq t \leq n}\left|\sigma_{t}^{-2}-\sigma_{0, t}^{-2}\right| \leq \max _{1 \leq t \leq n} \omega_{0} /\left(\pi_{0} x_{t-1}^{2}\right)^{2}=O_{p}\left(w_{n \xi}^{-4}\right)$. Thus, by the same arguments as in 
the proof of Lemma 8,

$$
\begin{aligned}
\frac{1}{\sigma_{t}^{2}} \frac{\partial \sigma_{t}^{2}(\vartheta)}{\partial \omega} & =\frac{1}{\sigma_{t}^{2}} \sum_{i=1}^{t} \beta^{i-1}=\frac{1}{\sigma_{0, t}^{2}} \frac{1}{1-\beta}+O_{p}\left(w_{n \xi}^{-4}\right), \\
\frac{1}{\sigma_{t}^{2}} \frac{\partial \sigma_{t}^{2}(\vartheta)}{\partial \alpha} & =\sum_{i=1}^{t} \beta^{i-1} \frac{y_{t-i}^{2}}{\sigma_{0, t}^{2}}+o_{p}(1)=\frac{\partial r_{t}^{*}(\theta)}{\partial \alpha}+o_{p}(1), \\
\frac{1}{\sigma_{t}^{2}} \frac{\partial \sigma_{t}^{2}\left(\vartheta_{0}\right)}{\partial \beta} & =\sum_{i=1}^{t} \beta^{i-1} \frac{\sigma_{t-i}^{2}(\vartheta)}{\sigma_{0, t}^{2}}+o_{p}(1)=\frac{\partial r_{t}^{*}(\theta)}{\partial \beta}+o_{p}(1), \\
\frac{1}{\sigma_{t}^{2}} \frac{\partial \sigma_{t}^{2}(\vartheta)}{\partial \pi} & =\sum_{i=1}^{t} \beta^{i-1} \frac{x_{t-i}^{2}}{\sigma_{0, t}^{2}}+o_{p}(1)=\frac{\partial r_{t}^{*}(\theta)}{\partial \pi}+o_{p}(1),
\end{aligned}
$$

uniformly in $t=1, \ldots, n$ and $\vartheta$, where $r_{t}^{*}(\theta)$ is defined in eq. (18). In total,

$$
\frac{\partial r_{t}(\vartheta)}{\partial \theta}=\frac{1}{\sigma_{t}^{2}} \frac{\partial \sigma_{t}^{2}(\vartheta)}{\partial \theta}=\frac{\partial r_{t}^{*}(\theta)}{\partial \theta}+o_{p}(1)
$$

It is easily seen that $\mathbb{E}\left[\sup _{\theta \in \Theta}\left\|\partial r_{t}^{*}(\theta) /(\partial \theta)\right\|^{2+\delta}\right]<\infty$ for some $\delta>0$ by the same arguments as in Lemma 8. Similarly, it is easily shown that

$$
\frac{1}{\sigma_{t}^{2}} \frac{\partial \sigma_{t}^{2}(\vartheta)}{\partial \omega \partial \beta}=-\frac{1}{\sigma_{0, t}^{2}} \frac{1}{(1-\beta)^{2}}+o_{p}(1), \quad \frac{\partial^{2} r_{t}(\vartheta)}{\partial \theta \partial \theta^{\prime}}=\frac{1}{\sigma_{t}^{2}} \frac{\partial^{2} \sigma_{t}^{2}(\vartheta)}{\partial \theta \partial \theta^{\prime}}=\frac{\partial^{2} r_{t}^{*}(\theta)}{\partial \theta \partial \theta^{\prime}}+o_{p}(1),
$$

where $\mathbb{E}\left[\sup _{\theta \in \Theta}\left\|\partial^{2} r_{t}^{*}(\theta) /\left(\partial \theta \partial \theta^{\prime}\right)\right\|\right]<\infty$, while $\partial \sigma_{t}^{2}(\vartheta) /\left(\partial \omega \partial \vartheta_{k}\right)=0, k=1,2,3$.

We now verify the conditions in Lemmas 11-12 of Kristensen and Rahbek (2010) which in turn imply local consistency and the claimed asymptotic distribution, respectively. To write our estimation problem in their notation, define $v_{\omega, n}=n^{1 / 4-d / 2}$ and $v_{\theta, n}=n^{1 / 2}$, so that $V_{n}$ defined in eq. (13) can be written as $V_{n}=\operatorname{diag}\left\{v_{\omega, n}, v_{\theta, n} I_{3}\right\}$. Next, we let $Q_{n}(\vartheta)=L_{n}(\vartheta) / v_{\omega, n}^{2}$ denote the normalized log-likelihood and let $U_{n}=V_{n} / v_{\omega, n}=\operatorname{diag}\left\{1, n^{1 / 4+d / 2} I_{3}\right\}$ be the associated rate matrix. We then claim that

$$
\text { (i) } v_{\omega, n} U_{n}^{-1} \frac{\partial Q_{n}\left(\vartheta_{0}\right)}{\partial \vartheta} \rightarrow{ }_{d} M N\left(0, \Sigma^{\mathrm{nst}}\right), \quad \text { (ii) }-U_{n}^{-1} \frac{\partial^{2} Q_{n}\left(\vartheta_{0}\right)}{\partial \vartheta \partial \vartheta^{\prime}} U_{n}^{-1} \rightarrow{ }_{p} H^{\mathrm{nst}}>0
$$

and, with $\mathcal{B}_{n}\left(\vartheta_{0}, \epsilon\right)=\left\{\vartheta:\left\|U_{n}\left(\vartheta-\vartheta_{0}\right)\right\|<\epsilon\right\}$ for some small $\epsilon>0$,

$$
\sup _{\vartheta \in \mathcal{B}_{n}\left(\vartheta_{0}, \epsilon\right)}\left\|U_{n}^{-1}\left\{\frac{\partial^{2} Q_{n}(\vartheta)}{\partial \vartheta \partial \vartheta}-\frac{\partial^{2} Q_{n}\left(\vartheta_{0}\right)}{\partial \vartheta \partial \vartheta}\right\} U_{n}^{-1}\right\|=O_{p}(\epsilon)
$$

Note that (i) of eq. (26) implies that $U_{n}^{-1} \partial Q_{n}\left(\vartheta_{0}\right) /(\partial \vartheta)=o_{p}(1)$. We first show (ii) of eq. (26): Note that

$$
U_{n}^{-1} \frac{\partial^{2} Q_{n}\left(\vartheta_{0}\right)}{\partial \vartheta \partial \vartheta^{\prime}} U_{n}^{-1}=\left\{v_{\omega, n} U_{n}\right\}^{-1} H_{n}\left(\vartheta_{0}\right)\left\{v_{\omega, n} U_{n}\right\}^{-1}=\left[\begin{array}{cc}
n^{d-1 / 2} H_{n, \omega \omega} & n^{d / 2-3 / 4} H_{n, \omega \theta} \\
n^{d / 2-3 / 4} H_{n, \theta \omega} & n^{-1} H_{n, \theta \theta}
\end{array}\right] .
$$


We analyze the four elements of $H_{n}\left(\vartheta_{0}\right)$ separately. First, using the above approximations, $h_{\theta \theta, t}(\vartheta)=$ $h_{\theta \theta, t}^{*}(\theta)+o_{p}(1)$ where

$$
h_{\theta \theta, t}^{*}(\theta):=\left\{\frac{\partial^{2} r_{t}^{*}(\theta)}{\partial \theta \partial \theta^{\prime}}-\frac{\partial r_{t}^{*}(\theta)}{\partial \theta} \frac{\partial r_{t}^{*}(\theta)}{\partial \theta^{\prime}}\right\}\left\{\frac{\varepsilon_{t}^{2}}{r_{t}^{*}(\theta)}-1\right\}-\frac{\partial r_{t}^{*}(\theta)}{\partial \theta} \frac{\partial r_{t}^{*}(\theta)}{\partial \theta^{\prime}} \frac{\varepsilon_{t}^{2}}{r_{t}^{*}(\theta)}
$$

The process $h_{\theta \theta, t}^{*}(\theta)$ is stationary and ergodic with $E\left[\sup _{\theta \in \Theta}\left\|h_{\theta \theta, t}^{*}(\theta)\right\|\right]<\infty$. It therefore follows from the uniform LLN that $\sup _{\vartheta}\left\|H_{n, \theta \theta}(\vartheta) / n-H_{\theta \theta}^{\mathrm{nst}}(\theta)\right\| \rightarrow_{p} 0$ where $H_{\theta \theta}^{\mathrm{nst}}(\theta)=\mathbb{E}\left[h_{\theta \theta, t}^{*}(\theta)\right]$. Next, using eq. (21),

$$
\begin{aligned}
-n^{d-1 / 2} H_{n, \omega \omega}\left(\vartheta_{0}\right) & =\frac{1}{\left(1-\beta_{0}\right)^{2}} \times \frac{1}{n^{1 / 2-d}} \sum_{t=1}^{n} \frac{2 \varepsilon_{t}^{2}-1}{\left(\omega_{0}+\pi_{0} x_{t-1}^{2}\right)^{2} z_{t}^{2}}+o_{p}(1) \\
& =\frac{1}{\left(1-\beta_{0}\right)^{2}} \times \frac{1}{n^{1 / 2-d}} \sum_{t=1}^{n} \frac{w_{t}}{\left(\omega_{0}+\pi_{0} x_{t-1}^{2}\right)^{2}}+o_{p}(1)
\end{aligned}
$$

where $w_{t}:=\left(2 \varepsilon_{t}^{2}-1\right) / z_{t}^{2}$ is stationary and geometrically $\beta$-mixing, c.f. Carrasco and Chen (2002). Since $w_{t}$ and $x_{t}$ are independent and $f(x)=1 /\left(\omega_{0}+\pi_{0} x^{2}\right)^{2}$ is integrable, we can employ Lemma $6(\mathrm{i})$ to obtain $-n^{d-1 / 2} H_{n, \omega \omega}\left(\vartheta_{0}\right) \rightarrow{ }_{d} H_{\omega \omega}^{\mathrm{nst}}$. Similarly,

$$
\begin{aligned}
-n^{d-1 / 2} H_{n, \omega \alpha}\left(\vartheta_{0}\right) & =\frac{1}{1-\beta} \times \frac{1}{n^{1 / 2}-d} \sum_{t=1}^{n} \frac{2 \varepsilon_{t}^{2}-1}{\left(\omega_{0}+\pi_{0} x_{t-1}^{2}\right) z_{t}} \frac{\partial r_{t}^{*}(\theta)}{\partial \alpha}+o_{p}(1) \\
& \rightarrow{ }_{d} K \times L_{W_{d}}(1,0) \int_{-\infty}^{\infty} \frac{1}{\omega_{0}+\pi_{0} s^{2}} d s \frac{1}{1-\beta_{0}} \mathbb{E}\left[\frac{\partial r_{t}^{*}(\theta)}{\partial \alpha} z_{t}^{-1}\right] .
\end{aligned}
$$

In particular, $n^{d / 2-3 / 4} H_{n, \omega \alpha}\left(\vartheta_{0}\right)=n^{-1 / 4-d / 2} \times\left\{n^{d-1 / 2} H_{n, \omega \alpha}\left(\vartheta_{0}\right)\right\}=o_{p}(1)$ since $-1 / 2<d<1 / 2$. The other cross-terms involving $\omega$ are shown to be $o_{p}(1)$ in the same manner. Next, we show (i) of eq. (26): Observe that $V_{n}^{-1} S_{n}\left(\vartheta_{0}\right)=\left[n^{d / 2-1 / 4} S_{n, \omega}\left(\vartheta_{0}\right), n^{-1 / 2} S_{n, \theta}\left(\vartheta_{0}\right)\right]^{\prime}$. It follows from Lemma $6\left(\right.$ ii) that $n^{d / 2-1 / 4} S_{n, \omega}\left(\vartheta_{0}\right) \rightarrow{ }_{d} M N\left(0, \Sigma_{\omega \omega}^{\text {nst }}\right)$ while, employing the same arguments as in the proof of Theorem 5 together with the stationary approximation results derived above, $n^{-1 / 2} S_{n, \theta}\left(\vartheta_{0}\right) \rightarrow_{d}$ $N\left(0, \Sigma_{\theta \theta}^{\mathrm{nst}}\right)$. The convergence is joint since the martingale difference, $\varepsilon_{t}^{2}-1$, is common to the two components of the score, and it is easily checked, by the same arguments as for the hessian, that $\Sigma_{\omega \theta}^{\mathrm{nst}}=O_{1 \times 3}$.

Finally, we verify eq. (27): We have already proved that this holds for $H_{n, \theta \theta}(\vartheta)$. What remains is to show that it also holds for the components involving $\omega$. We only show the result for $\partial^{2} Q_{n}(\vartheta) /\left(\partial \omega^{2}\right)$ since the proof for the other partial derivatives follows along the same lines. For $\vartheta \in \mathcal{B}_{n}\left(\vartheta_{0}, \epsilon\right),\left\|\theta-\theta_{0}\right\| \leq n^{-1 / 4-d / 2} \epsilon$ and $\left\|\omega-\omega_{0}\right\| \leq \epsilon$. Thus, by the mean-value theorem, for some $\bar{\vartheta}$ on the line segment connecting $\vartheta$ and $\vartheta_{0}$,

$$
\begin{aligned}
\left|\frac{\partial^{2} Q_{n}(\vartheta)}{\partial \omega^{2}}-\frac{\partial^{2} Q_{n}\left(\vartheta_{0}\right)}{\partial \omega^{2}}\right| & \leq\left\|n^{-1 / 2+d} \frac{\partial H_{n, \omega \omega}(\bar{\vartheta})}{\partial \theta}\right\|\left\|\theta-\theta_{0}\right\|+\left\|n^{-1 / 2+d} \frac{\partial H_{n, \omega \omega}(\bar{\vartheta})}{\partial \omega}\right\|\left\|\omega-\omega_{0}\right\| \\
& \leq\left\|n^{-3 / 4+d / 2} \frac{\partial H_{n, \omega \omega}(\bar{\vartheta})}{\partial \theta}\right\| \epsilon+\left\|n^{-1 / 2+d} \frac{\partial H_{n, \omega \omega}(\bar{\vartheta})}{\partial \omega}\right\| \epsilon .
\end{aligned}
$$


We then wish to show that $n^{-3 / 4+d / 2} \partial H_{n, \omega \omega}(\bar{\vartheta}) /(\partial \theta)=O_{p}(1)$ and $n^{-1 / 2+d} \partial H_{n, \omega \omega}(\bar{\vartheta}) /(\partial \omega)=$ $O_{p}(1)$. The third-order derivative is $\partial H_{n, \omega \omega}(\vartheta) /(\partial \vartheta)=\sum_{t=1}^{n} \partial h_{\omega \omega, t}(\vartheta) /(\partial \vartheta)$ where, using that $\partial^{2} \sigma_{t}^{2}(\vartheta) /\left(\partial \omega^{2}\right)=\partial^{3} \sigma_{t}^{2}(\vartheta) /\left(\partial \omega^{2} \partial \vartheta\right)=0$,

$$
\frac{h_{\omega \omega, t}(\vartheta)}{\partial \vartheta_{k}}=\frac{2}{\sigma_{t}^{6}(\vartheta)}\left(\frac{\partial \sigma_{t}^{2}(\vartheta)}{\partial \omega}\right)^{2} \frac{\partial \sigma_{t}^{2}(\vartheta)}{\partial \vartheta_{k}}\left\{\frac{\sigma_{t}^{2} \varepsilon_{t}^{2}}{\sigma_{t}^{2}(\vartheta)}-1\right\}+2\left(\frac{\partial \sigma_{t}^{2}(\vartheta)}{\partial \omega}\right)^{2} \frac{\sigma_{t}^{2} \varepsilon_{t}^{2}}{\sigma_{t}^{8}(\vartheta)} \frac{\partial \sigma_{t}^{2}(\vartheta)}{\partial \vartheta_{k}}
$$

As shown in the proof of Theorem $9, \sigma_{t}^{2} / \sigma_{t}^{2}(\vartheta) \leq W_{t}$ with $\mathbb{E}\left[W_{t}^{k}\right]<\infty$ for any $k>0$, and so

$$
\begin{aligned}
\left|\frac{h_{\omega \omega, t}(\vartheta)}{\partial \vartheta_{k}}\right| & \leq \frac{2}{\sigma_{t}^{6}(\vartheta)} \frac{1}{(1-\beta)^{2}}\left|\frac{\partial \sigma_{t}^{2}(\vartheta)}{\partial \vartheta_{k}}\right|\left\{W_{t} \varepsilon_{t}^{2}+1\right\}+2 \frac{1}{(1-\beta)^{2}} \frac{1}{\sigma_{t}^{6}(\vartheta)}\left|\frac{\partial \sigma_{t}^{2}(\vartheta)}{\partial \vartheta_{k}}\right| W_{t} \varepsilon_{t}^{2} \\
& \leq C \frac{1}{\sigma_{t}^{6}(\vartheta)}\left|\frac{\partial \sigma_{t}^{2}(\vartheta)}{\partial \vartheta_{k}}\right|\left\{W_{t} \varepsilon_{t}^{2}+1\right\} .
\end{aligned}
$$

Employing the same arguments as in the analysis of the hessian, we obtain the desired result.

Proof of Theorem 11. For both the stationary and non-stationary case, we have already shown as part of the proofs of Theorems 5 and 10 that $\sup _{\left\|U_{n}\left(\vartheta-\vartheta_{0}\right)\right\|<\delta}\left\|V_{n}^{-1} H_{n}(\vartheta) V_{n}^{-1}-H(\vartheta)\right\| \rightarrow p$. In the nonstationary case, $V_{n}$ is defined in eq. (13), $U_{n}=V_{n} / v_{\omega, n}$ and $H(\vartheta)=H^{\mathrm{nst}}(\vartheta)$; in the stationary case, $V_{n}=\sqrt{n} I_{4}, U_{n}=I_{4}$ and $H(\vartheta)=H^{\text {st }}(\vartheta)$. We now analyze $\hat{\Sigma}=\Sigma_{n}(\hat{\vartheta})$ where $\Sigma_{n}(\vartheta)=$ $\sum_{t=1}^{n} s_{t}(\vartheta) s_{t}(\vartheta)^{\prime}$ and $s_{t}(\vartheta)=\partial \ell_{t}(\vartheta) /(\partial \vartheta)$. First consider the stationary case: As part of the proof of Theorem 5, it was also shown that $s_{t}(\vartheta)=\sigma_{0, t}^{-2}(\vartheta) \partial \sigma_{0, t}^{2}(\vartheta) /(\partial \vartheta)\left\{y_{t}^{2} / \sigma_{0, t}^{2}(\vartheta)-1\right\}+o_{p}(1)$. The first term on the right hand side is continuous w.r.t. $\vartheta$ and, by Lemma 4 , is uniformly bounded by a stationary sequence with second moment. It therefore follows by the uniform LLN, that $\sup _{\left\|\vartheta-\vartheta_{0}\right\|<\delta}\left\|\Sigma_{n}(\vartheta) / n-\Sigma^{\text {st }}(\vartheta)\right\| \rightarrow p 0$ where $\vartheta \mapsto \Sigma^{\text {st }}(\vartheta)$ is continuous; in particular, $\Sigma_{n}(\hat{\vartheta}) / n \rightarrow p$ $\Sigma^{\text {st }}$. In conclusion, $n \hat{\Omega} \rightarrow p \Omega^{\text {st }}$ and so $\hat{\Omega}^{-1 / 2}\left\{\hat{\vartheta}-\vartheta_{0}\right\}=(\hat{\Omega} / n)^{-1 / 2} \sqrt{n}\left\{\hat{\vartheta}-\vartheta_{0}\right\} \rightarrow_{d} N\left(0, I_{4}\right)$.

For the non-stationary case, we proceed as in the analysis of the hessian: First, write

$$
\Sigma_{n}(\vartheta)=\left[\begin{array}{cc}
\Sigma_{n, \omega \omega}(\vartheta) & \Sigma_{n, \omega \theta}(\vartheta) \\
\Sigma_{n, \theta \omega}(\vartheta) & \Sigma_{n, \theta \theta}(\vartheta)
\end{array}\right]=\sum_{t=1}^{n}\left[\begin{array}{cc}
s_{t, \omega}^{2}(\vartheta) & s_{t, \omega}(\vartheta) s_{t, \theta}(\vartheta)^{\prime} \\
s_{t, \omega}(\vartheta) s_{t, \theta}(\vartheta) & s_{t, \theta}(\vartheta) s_{t, \theta}(\vartheta)^{\prime}
\end{array}\right]
$$

where $s_{t, \omega}(\vartheta)$ and $s_{t, \theta}(\vartheta)$ denote the partial derivatives of $\ell_{t}(\vartheta)$ w.r.t. $\omega$ and $\theta$, respectively. Observe that $s_{t, \theta}(\vartheta)$ has a stationary approximation, and so, similar to the stationary case, we can appeal to a uniform LLN for stationary and ergodic sequences to obtain $\Sigma_{n, \theta \theta}(\hat{\vartheta}) / n \rightarrow p \Sigma^{\mathrm{nst}}$. Next,

$$
n^{d-1 / 2} \sum_{t=1}^{n} s_{t, \omega}^{2}\left(\vartheta_{0}\right)=\frac{1}{\left(1-\beta_{0}\right)^{2}} \times n^{d-1 / 2} \sum_{t=1}^{n} \frac{1}{\left(\omega_{0}+\pi_{0} x_{t-1}^{2}\right)^{2} z_{t}^{2}}\left\{\varepsilon_{t}^{2}-1\right\}^{2}+o_{p}(1) \rightarrow_{d} \Sigma_{\omega \omega}^{\mathrm{nst}}
$$

and, similar to the proof of eq. (27), $\sup _{\left\|U_{n}\left(\vartheta-\vartheta_{0}\right)\right\|<\delta}\left\|n^{d-1 / 2} \sum_{t=1}^{n}\left\{s_{t, \omega}^{2}(\vartheta)-s_{t, \omega}^{2}\left(\vartheta_{0}\right)\right\}\right\|=o_{p}(1)$. Similarly, we can show that $n^{d / 2-3 / 4} \sum_{t=1}^{n} s_{t, \omega}(\vartheta) s_{t, \theta}(\vartheta)^{\prime}=o_{p}(1)$. In conclusion, $V_{n}^{-1} \hat{\Omega} V_{n}^{-1} \rightarrow_{p} \Omega^{\text {st }}$ and so $\hat{\Omega}^{-1 / 2}\left\{\hat{\vartheta}-\vartheta_{0}\right\}=\left(V_{n}^{-1} \hat{\Omega} V_{n}^{-1}\right)^{-1 / 2} V_{n}\left\{\hat{\vartheta}-\vartheta_{0}\right\} \rightarrow{ }_{d} N\left(0, I_{4}\right)$. 


\section{References}

Barndorff-Nielsen, O.E. and Shephard, N. (2007), "Variation, jumps and high frequency data in financial econometrics," in Advances in Economics and Econometrics. Theory and Applications, Ninth World Congress, R. Blundell, T. Persson and W.K. Newey (eds.), Econometric Society Monographs, Cambridge University Press, pp. 328-372.

Berkes, I., L. Horváth and P. Kokoszka (2003), "GARCH processes: Structure and estimation," Bernoulli 9(2), 201-227.

Billingsley, P. (1974), "Conditional distributions and tightness," Annals of Probability 2, 480-485.

Bollerslev, T. (1986), "Generalized autoregressive conditional heteroskedasticity," Journal of Econometrics 31, 307-327.

Bollerslev, T. and M. Melvin (1994), "Bid-Ask spreads and the volatility in the foreign exchange market: An empirical analysis," Journal of International Economics 36, 355-372.

Brandt, A. (1986), "The stochastic equation $Y_{n+1}=A_{n} Y_{n}+B_{n}$ with stationary coefficients," Advances in Applied Probability 18, 211-220.

Brenner, R.J., R.H. Harjes and K.F. Kroner (1996), "Another look at models of the short-term interest rate," Journal of Financial and Quantitative Analysis 31, 85-107.

Brown, B. (1971), "Martingale central limit theorems," Annals of Mathematical Statistics 42, 59-66.

Carrasco, M. and X. Chen (2002), "Mixing and moment properties of various GARCH and stochastic volatility models," Econometric Theory 18, 17-39.

Dittmann, I. and C.W.J. Granger (2002), "Properties of nonlinear transformations of fractionally integrated processes," Journal of Econometrics 110, 113-133.

Dominguez, K. (1998), "Central bank intervention and exchange rate volatility," Journal of International Money and Finance 17, 161-190.

Engle, R.F. (2002), "New frontiers for ARCH models," Journal of Applied Econometrics, 17, 425446.

Engle, R.F. and G.M. Gallo (2006), "A multiple indicators model for volatility using intra-daily data," Journal of Econometrics, 131, 3-27.

Escanciano, J.M. (2009), "Quasi-maximum likelihood estimation of semi-strong GARCH models," Econometric Theory 25, 561-570.

Fleming, J., C. Kirby and B. Ostdiek (2008), "The specification of GARCH models with stochastic covariates," Journal of Futures Markets, 28, 911-934. 
Franq, C. and J.-M. Zakoïan (2004), "Maximum likelihood estimation of pure GARCH and ARMAGARCH processes," Bernoulli 10, 605-637.

Franq, C. and J.-M. Zakoïan (2012), "Strict stationarity testing and estimation of explosive and stationary generalized autoregressive conditional heteroskedasticity models," Econometrica 80, $821-861$.

Gallo, G., and B. Pacini (2000), "The effects of trading activity on market volatility," European Journal of Finance 6, 163-175.

Girma, P., and M. Mougoue (2002), "An empirical examination of the relation between futures spreads volatility, volume, and open interest," Journal of Futures Markets 22, 1083-1102.

Hagiwara, M., and M. Herce (1999), "Endogenous exchange rate volatility, trading volume and interest rate differentials in a model of portfolio selection," Review of International Economics 7, 202-218.

Hall, P. and C.C. Heyde (1980), "Martingale Limit Theory and Its Application. New York: Academic Press.

Han, H. (2014), "Asymptotic properties of GARCH-X processes," forthcoming in Journal of Financial Econometrics.

Han, H. and J.Y. Park (2012), "ARCH/GARCH with persistent covariates: Asymptotic theory of MLE," Journal of Econometrics 167, 95-112.

Han, H. and J.Y. Park (2013), "GARCH with omitted persistent covariate," unpublished manuscript, Kyung Hee University.

Hansen, P.R., Z. Huang and H.H. Shek (2012), "Realized GARCH: A complete model of returns and realized measures of volatility," Journal of Applied Econometrics 27, 877-906.

Hodrick, R. (1989), "Risk, uncertainty, and exchange rates," Journal of Monetary Economics, 23, 433-459.

Hwang, S. and S.E. Satchell (2005), "GARCH Model with cross-sectional volatility: GARCH-X models," Applied Financial Economics 15, 203-216.

Heber, G., A. Lunde, N. Shephard and K. Sheppard (2009), OMI's realised library, Version 0.1, Oxford-Man Institute, University of Oxford.

Jensen, S.T. and A. Rahbek (2004), "Asymptotic inference for nonstationary GARCH," Econometric Theory, 20, 1203-1226.

Kasparis, I., E. Andreou and P.C.B. Phillips (2012), "Nonparametric predictive regression," unpublished manuscript, University of Cyprus. 
Kristensen, D. and A. Rahbek (2005), "Asymptotics of the QMLE for a class of $\operatorname{ARCH}(q)$ models," Econometric Theory 21, 946-961.

Kristensen, D. and A. Rahbek (2010), "Likelihood-based inference for cointegration with nonlinear error-correction," Journal of Econometrics 158, 78-94.

Kristensen, D. and Y. Shin (2012), "Estimation of dynamic models with nonparametric simulated maximum likelihood," Journal of Econometrics 167, 76-94.

Lamoureux, C. G. and W. D. Lastrapes (1990), "Heteroskedasticity in stock return data: Volume versus GARCH effects," Journal of Finance 45, 221-229.

Marsh, T. A., and N. Wagner (2005), "Surprise volume and heteroskedasticity in equity market returns," Quantitative Finance, 5, 153-168.

Park, J.Y. and P.C.B. Phillips (1999), "Asymptotics for nonlinear transformations of integrated time series," Econometric Theory, 15, 269-298.

Park, J.Y. and P.C.B. Phillips (2001), "Nonlinear regressions with integrated time series," Econometrica, 69, 117-161.

Shephard, N. and K. Sheppard (2010), "Realising the future: forecasting with high frequency based volatility (HEAVY) models," Journal of Applied Econometrics 25, 197-231.

Wang, Q. (2013), "Martingale limit theorem revisited and nonlinear cointegrating regression," forthcoming in Econometric Theory.

Wang, Q. and P.C.B. Phillips (2009a), "Asymptotic theory for local time density estimation and nonparametric cointegrating regression," Econometric Theory 25, 710-738.

Wang, Q. and P.C.B. Phillips (2009b), "Structural nonparametric cointegrating regression," Econometrica $77,1901-1948$.

Yoshihara K. (1976), "Limiting behaviour of U-statistics for stationary, absolutely regular processes," Zeitschrift für Wahrscheinlichkeitstheorie und verwandte Gebiete 35, 237-252. 